\title{
Non-invasive in vivo optical monitoring of human placental oxygenation
}

\author{
Lin Wang ( $\square$ linwang3@sas.upenn.edu ) \\ University of Pennsylvania \\ Jeffrey Cochran \\ University of Pennsylvania

\section{Tiffany Ko} \\ Children's Hospital of Philadelphia

\section{Wesley Baker} \\ Children's Hospital of Philadelphia

\section{Kenneth Abramson} \\ University of Pennsylvania

\section{Lian $\mathrm{He}$} \\ University of Pennsylvania

\section{David Busch} \\ University of Texas
}

\section{Venki Kavuri}

University of Pennsylvania

Rebecca Linn

Children's Hospital of Philadelphia https://orcid.org/0000-0001-7671-4681

\section{Samuel Parry}

University of Pennsylvania

\section{Arjun Yodh}

University of Pennsylvania https://orcid.org/0000-0003-4744-2706

\section{Nadav Schwartz}

University of Pennsylvania

\section{Article}

Keywords: placental blood oxygenation, Frequency Domain Diffuse Optical Spectroscopy, ultrasound Posted Date: January 14th, 2021

DOI: https://doi.org/10.21203/rs.3.rs-139102/v1 
License: (c) (i) This work is licensed under a Creative Commons Attribution 4.0 International License. Read Full License

Version of Record: A version of this preprint was published at Nature Biomedical Engineering on August 15th, 2022. See the published version at https://doi.org/10.1038/s41551-022-00913-2. 


\section{Non-invasive in vivo optical monitoring of human placental oxygenation}

2 Lin Wang ${ }^{1 *}$, Jeffrey M. Cochran ${ }^{1}$, Tiffany Ko ${ }^{1,3}$, Wesley B. Baker ${ }^{1,3}$, Kenneth Abramson ${ }^{1}$, Lian He ${ }^{1}$,

3 David R. Busch ${ }^{1,4}$, Venki Kavuri ${ }^{1}$, Rebecca L. Linn ${ }^{5}$, Samuel Parry ${ }^{2}$, Arjun G. Yodh ${ }^{1}$ and Nadav Schwartz ${ }^{2}$

$4 \quad{ }^{1}$ Department of Physics and Astronomy, University of Pennsylvania.

$5 \quad{ }^{2}$ Maternal and Child Health Research Center, Perelman School of Medicine, University of Pennsylvania.

$6 \quad$ 3Division of Neurology, Children's Hospital of Philadelphia.

$7 \quad{ }^{4}$ Southwestern Medical Center, University of Texas.

$8 \quad{ }^{5}$ Division of Anatomic Pathology, Children's Hospital of Philadelphia.

$9 \quad$ *E-mail: linwang3@sas.upenn.edu 
Direct assessment of human placental blood oxygenation can provide valuable information about placental function and, potentially, detect dysfunction. Currently however, no bedside tools exist for noninvasive monitoring of placental oxygenation. Here we report a continuous, non-invasive in vivo method to probe placental oxygen hemodynamics using deep penetrating Frequency Domain Diffuse Optical Spectroscopy (FD-DOS) with concurrent ultrasound (US) imaging. This multi-modal instrument facilitates assessment of placental oxygenation properties from image reconstruction algorithms that integrate anatomical US information about layer morphology with information from optics about functional hemodynamics. Tissue phantom experiments, simulations, and human subject studies validate the approach and demonstrate sensitivity to placental tissue located $\leq 5 \mathrm{~cm}$ below the surface. In a pilot study $(n=24)$, human placental oxygen hemodynamics are measured non-invasively during maternal hyperoxia. Initial results suggest placental response to maternal hyperoxia may serve as a tool to detect placenta-related adverse pregnancy outcome and maternal vascular malperfusion of placenta, weeks before delivery.

Abnormal placental development is widely accepted as the cause of common adverse pregnancy outcomes (APOs) such as hypertensive disorders, fetal growth restriction, and stillbirth. Moreover, placental dysfunction has been associated with morbidities in offspring, including perinatal mortality and long-term neurodevelopmental and cardiovascular consequences $^{1-3}$. To better understand human placental dysfunction associated with these APOs, non-invasive methods that measure placental oxygen dynamics are needed. Ultrasound (US) imaging is the primary clinical modality used for assessing pregnancy; it can provide indirect information about flow resistance in large blood vessels but is used primarily to derive morphological information. Thus, clinical US guidelines do not include direct functional assessment of the placenta ${ }^{4}$. Current knowledge about placental biology has been gleaned largely from ex-vivo tissue and from animal research ${ }^{5,6}$, which have limitations as models for dynamic changes in placental pathophysiology during pregnancy ${ }^{7,8}$. Similarly, the reference literature on placental oxygenation is derived largely from decades-old sheep studies, and from scant human data using invasive sampling techniques that have yielded varying results ${ }^{7,9}$. Lastly, even MRI tools for assessing placental oxygenation ${ }^{10,11}$ are poorly suited for bedside monitoring and generally rely on indirect signals $\mathbf{s}^{12}$. 
Here we demonstrate a novel instrument and methodology, based on diffuse optical spectroscopy (DOS) and ultrasound (US), which facilitates measurement of oxygen hemodynamics in complex organs such as the placenta, buried far below the tissue surface amidst intervening layered tissues. DOS measures oxy- and deoxyhemoglobin concentration; it has been successfully employed for assessment of tissue hemodynamics ${ }^{13-21}$ in clinical problems ranging from breast cancer diagnosis and therapy monitoring ${ }^{19,22}$, brain function ${ }^{23}$ and injury monitoring ${ }^{17}$. In most of these applications, however, tissues were studied with reflected light, and therefore light penetration was limited to less than $2 \mathrm{~cm}$ below the surface ${ }^{24,25}$. Our new instrumentation and algorithms provide the substantial improvements in methodology needed to measure oxygen hemodynamics of the anterior placenta, located as deep as $5 \mathrm{~cm}$ below the tissue surface, amidst intervening heterogeneous tissue layers. These improvements enable continuous functional studies of placenta at the bedside and create opportunities for investigation of hemodynamics in other organs located far below tissue surfaces.

Previously, Continuous-Wave Diffuse Optical Spectroscopy (CW-DOS) was explored for noninvasive measurement of placental blood oxygenation ${ }^{26,27}$. Unfortunately, these measurements utilized short sourcedetector separations $(\sim 4 \mathrm{~cm})$ on the tissue surface, which limited light depth penetration to $\sim 2 \mathrm{~cm}$. Furthermore, the $\mathrm{CW}$ approach requires major assumptions about tissue homogeneity and scattering that prevent quantification of absolute oxy- and deoxy-hemoglobin concentrations ${ }^{28}$ and do not account for the layered morphology of the abdomen. Thus, early progress with placenta optical studies was limited in measurement accuracy and prognostic value. Our work benefits from more accurate implementation of light transport models ${ }^{13-15,29}$, and from more sophisticated Frequency- and Time-Domain (FD-DOS, TD-DOS) optical instrumentation advances that permit relaxation of assumptions about tissue scattering and homogeneity. The resulting custom, state-of-the-art FDDOS instrument advances the optical measurement frontier. It has signal-to-noise ratio (SNR) sufficient to permit source-detector separations (SDSs) as large as $10 \mathrm{~cm}$ on the tissue surface, enabling light penetration of $~ 5 \mathrm{~cm}$ and improving upon previous SDS records ${ }^{30-32}$. Importantly, the optical instrument is integrated with US imaging in the same probe head ${ }^{22}$. This multi-modal FD-DOS/US combination facilitates integration of anatomic US information about tissue layer morphology with functional hemodynamic information about deep tissues from FDDOS. The anatomic information enables tissue-specific, layered image reconstruction which separates the hemodynamic properties and responses of deep tissues, such as placenta, from those of overlying layers.

We validated the new methodology in layered tissue phantoms and demonstrated its feasibility and utility by 
1 direct in vivo assessment of human placental oxygenation $(n=24)$. Specifically, we measured placental oxy-

$2\left[\mathrm{HbO}_{2}\right]$ and deoxy-hemoglobin $[\mathrm{Hb}]$ concentrations, or equivalently, total hemoglobin $\left[\mathrm{Hb}_{\mathrm{T}}\right]$ concentration and oxygen saturation $\left(\mathrm{StO}_{2}\right)$. We performed reproducibility and stability tests to characterize the technology, collected average tissue properties from each patient, and demonstrated detection of dynamic changes in placental oxygenation by varying maternal position and by performing maternal hyperoxia experiments. Notably, our pilot study shows that placental oxygen hemodynamics during maternal hyperoxia is significantly associated with placenta-related adverse pregnancy outcome (APO) and with placental maternal vascular malperfusion (MVM), a primary histopathologic pattern characteristic of placental dysfunction strongly associated with APO and with risk of long-term disease ${ }^{33,34}$. The results demonstrate potential for non-invasive detection of placental dysfunction and for generating improved clinical understanding of placental pathophysiology in vivo.

\section{Results}

Deep penetration-depth FD-DOS Instrumentation. To measure placental oxygen hemodynamics in vivo, we built an ultra-low-noise heterodyne instrument for Frequency-Domain Diffuse Optical Spectroscopy (FD-DOS). The instrument has sufficient dynamic range and SNR to perform FD-DOS measurements accurately at sourcedetector separations (SDSs) of $10 \mathrm{~cm}$. These capabilities permit quantitative determination of oxy- $\left[\mathrm{HbO}_{2}\right]$, deoxy$[\mathrm{Hb}]$, and total-hemoglobin $\left[\mathrm{Hb}_{T}\right]$ concentrations, and oxygen saturation $\left(\mathrm{StO}_{2}\right)$ in placenta located $\sim 5 \mathrm{~cm}$ below the skin surface. Fig. 1 (a) shows key features of the custom heterodyne FD-DOS instrument. It employs three lasers with wavelengths of $785,808,830 \mathrm{~nm}$. The output of each laser is radio frequency (RF) amplitudemodulated at $f_{1}=100 \mathrm{MHz}$. A critical new technical feature of the instrument is its exceptional laser modulation depth. To achieve this improvement, we divided the source driver signal into four sub-signals, amplified each subsignal in multiple stages with low-noise linear amplifiers, and then recombined and impedance-matched the amplified sub-signals for input to the laser drivers. Each laser's RF driver power was individually optimized to achieve $>90 \%$ light modulation depth, thereby increasing modulated diffusive wave amplitude and decreasing (unmodulated) background diffuse light. As a result, measurement SNR is significantly better than previous work (e.g., by $>20 \mathrm{~dB}$ for SDS $=8 \mathrm{~cm})$, enabling long SDS measurements with smaller input powers $(\sim 35 \mathrm{~mW})$. 
(a) Schematic of hybrid FD-DOS/US placenta instrumentation system

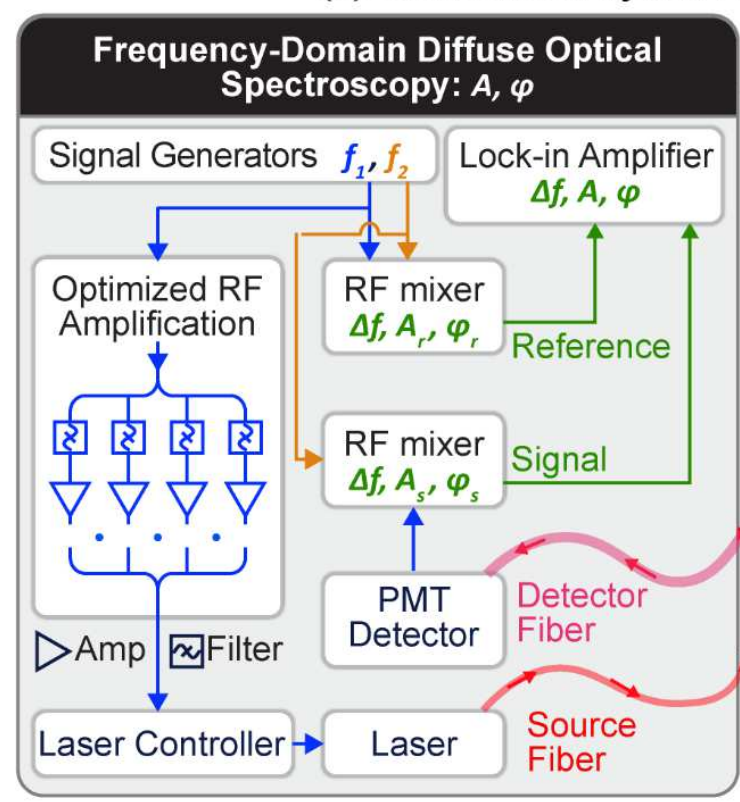

(b) Integration of optical and US instrumentation into single probe head
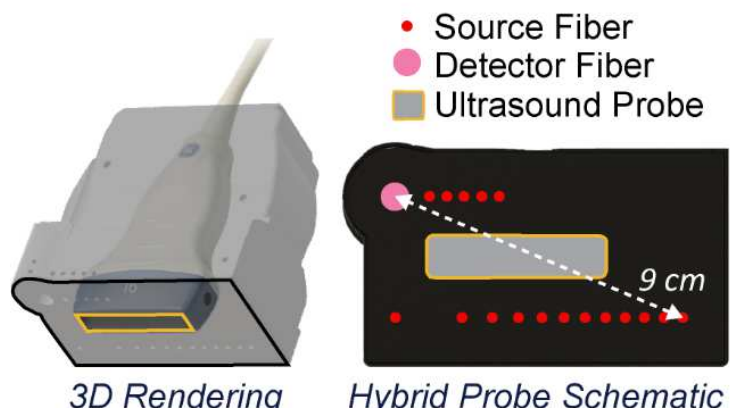

Hybrid Probe Schematic
Optical Reconstruction of Placental $\mathrm{StO}_{2},\left[\mathrm{Hb}_{T}\right],\left[\mathrm{HbO}_{2}\right]$
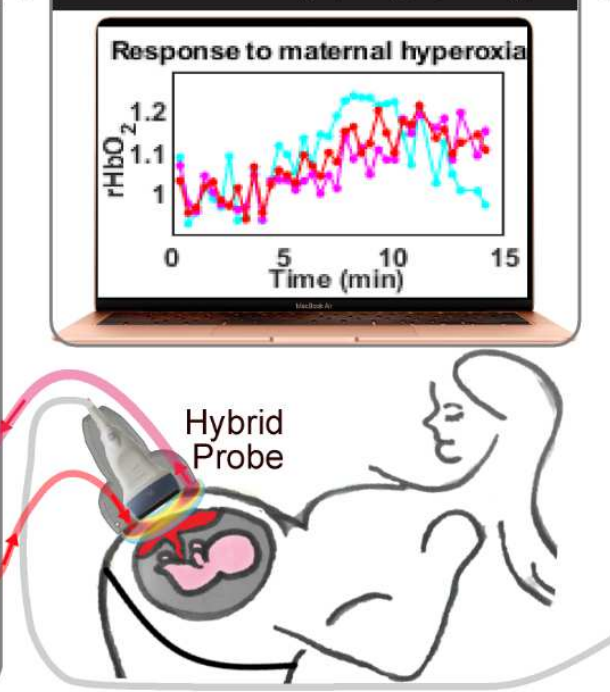

Ultrasound Image Segmentation: $d_{1}, d_{2}, d_{3}$

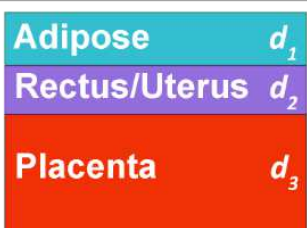

(c) US image segmentation and corresponding three-layer model for optical image reconstruction
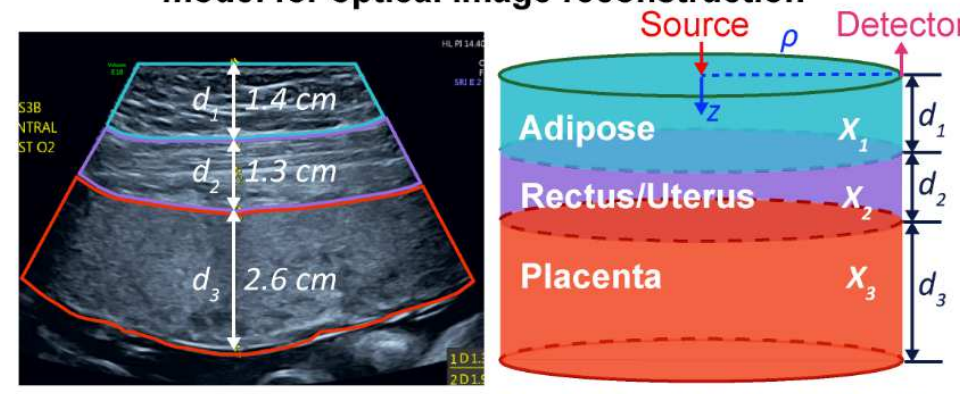

$X_{i}=\left\{\mathrm{StO}_{2, i}\left[\mathrm{Hb}_{T, i}\right],\left[\mathrm{HbO}_{2, i}\right]\right\}$

Fig. 1: Integrated Frequency-Domain Diffuse Optical Spectroscopy/Ultrasound (FD-DOS/US) placenta

instrumentation and three-layer modeling. (a) Schematic of the hybrid FD-DOS/US instrumentation showing laser amplitude modulation electronics, tissue light transmission, PMT detection, heterodyne mixing for frequency down-conversion, lock-in detection of light wave amplitude/phase, simultaneous US image segmentation, and optical image reconstruction of placental hemoglobin properties. (b) 3D rendering and bottom-view of probe.

$$
\text { These optoelectronics are fiber-coupled into a custom optical probe head, within which a commercial US probe }
$$
is also mounted. Optical source fibers in this probe offer 17 SDSs for patient measurements ranging, in the present case, between 1 and $9 \mathrm{~cm}$ (Fig. 1 (b)). During patient measurements, 10 source fiber locations are chosen to optimize coverage over the anatomic regions of interest for each patient, and we scan sequentially 
through them. At the end of each cycle, a dark count measurement is obtained to correct for systemic noise. A high-transmission $5 \mathrm{~mm}$ core diameter liquid light guide (detector fiber) collects and directs light to a photomultiplier tube (PMT) detector. The PMT converted electrical signal is mixed with another RF wave at $f_{2}=$ 100.2 $\mathrm{MHz}$ to generate heterodyne down-converted signals $\left(\Delta f=f_{1}-f_{2}=0.2 \mathrm{MHz}\right.$ ). A high-sampling-rate lock-in amplifier captures the amplitude and phase of the diffuse light waves.

Concurrent optical and ultrasound imaging. Custom integration of optical instrumentation with a commercial US system (9L-D probe, Voluson E10, GE Healthcare) provides another substantial technical improvement over prior placenta work. The custom probe facilitates concurrent measurement of tissue layer morphology and tissue physiological properties. This concept has been employed in breast cancer research ${ }^{22}$, but much deeper light penetration is required for placenta. The US transducer at the probe's center generates images (Fig. 1 (c)) which we utilize to segment target tissue into distinct layers that constrain optical reconstruction algorithms.

Three-layer modeling and reconstruction of placental properties. A critical algorithmic advance compared to prior placenta research is our use of tissue layer morphology from US imaging to constrain the photon diffusion tomographic inverse problem. In practice, we model the abdomen as three-layers: adipose, rectus/uterus, and placental tissue. We approximate each layer as homogeneous and laterally infinite, but with thickness and depth determined by US (Fig. 1 (c)).

The measurement geometry and modeling are shown in Fig. 1 (c). $\rho$ is the SDS on the tissue surface. Given the measured tissue layer thickness, as well as optical and physiological properties for each layer, standard methods $^{35,36}$ are employed to solve the diffusion equation and generate predictions for the detected light fluence rate on the tissue surface. Fig. 2(a) outlines our three-step reconstruction procedure (details provided in Methods). Briefly, each step of the three-step reconstruction finds "best" tissue properties by minimizing the difference between measured data and the predictions of diffuse optical tissue models of increasing complexity.

The STEP 1 assumes the underlying tissue is semi-infinite and homogeneous and employs the simplest analytic model for optical property reconstruction. The STEP 2 utilizes estimates from step one as initial guesses in a two-layer analytic diffuse optical tissue model. The STEP 3 utilizes estimates from step two as initial guesses in a three-layer diffuse optical tissue model. In all steps, layer thicknesses are fixed by US imaging, but other tissue properties are permitted to vary to find best estimates of tissue physiological and optical properties. 
(a) Three-layer optical image reconstruction protocol

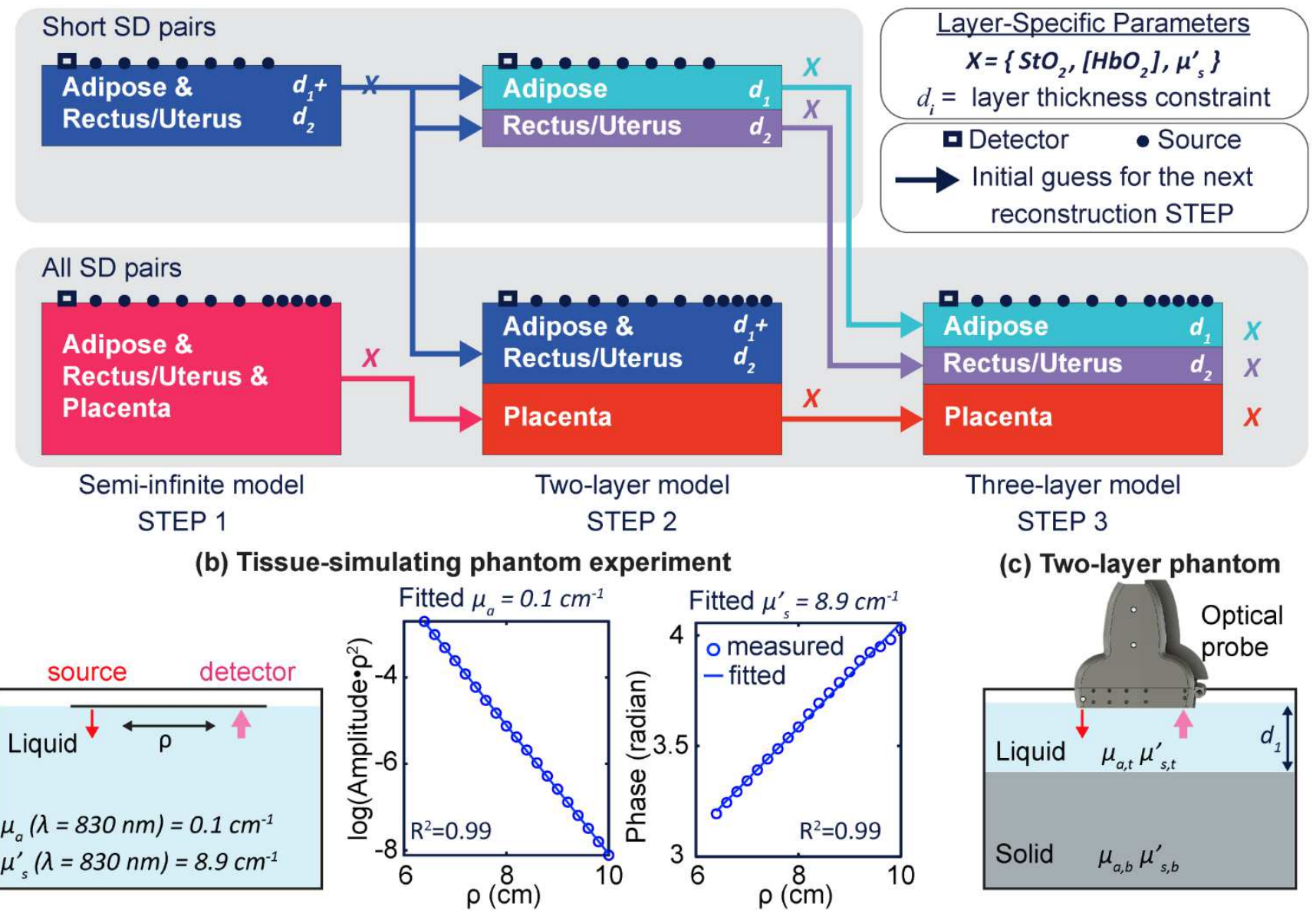

Fig. 2: Three-layer model reconstruction algorithm and phantom validation experiments. (a) Three-layer model initialization and reconstruction procedure. (b) Tissue-simulating phantom experiment for validating accuracy and depth sensitivity. A translation stage moves the source fiber across the phantom for measurements at SDSs ranging from 6.2 to $10 \mathrm{~cm}$. Nonlinear semi-infinite fitting is performed for each wavelength to reconstruct phantom optical properties. (c) Two-layer phantom experiment schematic.

Importantly, the image reconstructions rely on simultaneous fitting of data from all SDSs and all wavelengths. This multi-spectral, multi-SDS approach builds global constraints directly into the inverse problem and is critical for robust fitting. To avoid reconstruction overfitting, Tikhonov regularization is employed to reduce ill-posedness of the inverse problem. (Fitting/regularization details are in Methods.)

Experimental validation/characterization with tissue-simulating phantoms. We first characterized FD-DOS instrument performance using a tissue-simulating liquid phantom comprised ink for absorption, and $20 \%$ Intralipid 
(Baxter) for scattering (Fig. 2 (b)). In the first study, fittings based on semi-infinite homogeneous solutions of the diffusion equation were employed to reconstruct phantom optical properties (i.e., absorption coefficient $\left(\mu_{a}\left(\mathrm{~cm}^{-1}\right)\right)$, reduced scattering coefficient $\left.\mu_{s}^{\prime}\left(\mathrm{cm}^{-1}\right)\right)^{13}$. The data quality demonstrated good SNR at SDSs up to $10 \mathrm{~cm}$ (Fig. 2 (b)). Reconstructed optical properties at each wavelength had accuracies ranging from 3\% to $9 \%$.

In two-layer phantom experiments, a solid phantom with fixed optical properties was positioned in a liquid phantom, and the optical probe was set on the liquid surface (Fig. 2 (c)). An absorption-titration experiment evaluated instrument sensitivity, holding the over-layer liquid phantom thickness $(3 \mathrm{~cm})$ constant, while incrementally increasing the absorption coefficient of the top layer. A depth-changing experiment tested sensitivity to superficial layer thickness; here, the liquid phantom had fixed optical properties, and the superficial layer thickness was increased from 1.5 to $3.0 \mathrm{~cm}$. Experimental results demonstrated the instrument can extract deep layer tissue optical properties accurately across all of these conditions (details in Methods).

Validation of three-layer reconstruction with finite element simulations. We generated simulated data using a finite element simulation tool (TOAST) ${ }^{37}$ that facilitated creation of a three-layer model with segmented optical properties based on the layer morphology extracted from a patient ultrasound image (Fig. 1 (c)). Since patient layer interfaces are curved, we generated test data from curved patient layer interfaces (Fig. 1 (c) left). For the inverse problem, however, we assumed each layer interface to be flat (Fig. 1 (c) right). The three-layer reconstruction produced good estimates of $\mathrm{StO}_{2}$ and $\left[\mathrm{Hb}_{\mathrm{T}}\right]$ with $<10 \%$ error in the second and third layers (see Extended Data Table 2). The first layer $\left[\mathrm{Hb}_{T}\right]$ had a comparatively larger reconstruction error due to artificially large differences in simulated $\left[\mathrm{Hb}_{T}\right]$ concentrations between first and second layers; in real tissue, differences are smaller. We also found a relatively large error in the reconstructed scattering coefficient of the third layer; importantly, a theoretical analysis showed that this scattering uncertainty does not strongly affect reconstructed chromophore concentrations of the third layer.

In vivo placental oxygen dynamics. We performed a pilot clinical study of human placental oxygen-related hemodynamic properties (see Methods for Study Design). The study enrolled women with singleton pregnancies in their third trimester and with anterior placentas. The central region of placenta was targeted for monitoring. Adipose, rectus/uterus, and placenta layers were characterized by US and FD-DOS.

Four experiments were conducted: (1) a reproducibility experiment $(n=19) ;(2)$ a stability experiment $(n=24) ;(3)$ 
1 a maternal left tilt experiment $(n=3) ;(4)$ a maternal hyperoxia experiment $(n=24)$. The subjects in the hyperoxia study had a median (interquartile range (IQR)) gestational age of $34.5(32.9,35.4)$ weeks and Pre-gravid BMI of 27.7 (24.6, 30.2). The median (IQR) depth of the anterior placentas was $2.7(2.3,3.3) \mathrm{cm}$ beneath the skin surface, and the nulliparity of the subjects was $29 \%$. The subjects in the maternal tilt study had gestational age of $37.0(35.1,37.3)$ weeks and Pre-gravid BMI of $30.4(29.2,31.7)$. The median (IQR) depth of the anterior placentas was $2.3(1.9,2.4) \mathrm{cm}$ beneath the skin surface.

Reproducibility was assessed by calculating the Intra-class Correlation Coefficient (ICC) for 3 repeated measurements at the same placental location in 19 subjects. Placental $\mathrm{StO}_{2},\left[\mathrm{Hb}_{\mathrm{T}}\right]$, and $\left[\mathrm{HbO}_{2}\right]$ were highly repeatable within subjects (ICC $\geq 0.9)$.

Stability was assessed from the standard deviation (S.D.) of data derived during a continuous 10 -frame (3.5 minute) baseline measurement at the same location on each subject. This single subject S.D. was then averaged over the 24 subjects to derive a mean measurement S.D., which served as our stability criteria. The resulting mean S.D. of placental $\mathrm{StO}_{2},\left[\mathrm{Hb}_{\mathrm{T}}\right]$ and $\left[\mathrm{HbO}_{2}\right]$ were small, i.e.,2.1\%, $0.8 \mu \mathrm{M}$, and $0.7 \mu \mathrm{M}$, respectively; opticallyderived hemodynamic properties are stable at baseline (see Extended Data Fig. 2 (a)).

The maternal left tilt experiment is sensitive to positional changes in cardiac output and utero-placental perfusion that can lead to an increase in maternal cardiac output of up to $20 \%^{38}$. We measured placental hemoglobin properties of 3 subjects in the supine position and then had them tilt to the left lateral position without removing the probe. The averaged relative increase of $\mathrm{StO}_{2},\left[\mathrm{Hb}_{\mathrm{T}}\right]$ and $\left[\mathrm{HbO}_{2}\right]$ was $2.4 \%, 8.4 \%$, and $10.9 \%$, respectively. Mean values of $\mathrm{StO}_{2},\left[\mathrm{Hb}_{\mathrm{T}}\right]$, and $\left[\mathrm{HbO}_{2}\right]$ for each subject before and after the maternal tilt are presented in Extended Data Fig. 2 (b). Although the subject number is very small, we observed a trend; $\left[H b_{T}\right]$ and $\left[\mathrm{HbO}_{2}\right]$ increased in the lateral position $(\mathrm{P}=0.046, \mathrm{P}=0.018$, respectively), suggestive of an accompanying increase in placental perfusion with oxygenated maternal blood.

Finally, we measured placental hemodynamic response to maternal hyperoxia. Subjects were given $100 \% \mathrm{FiO}_{2}$ via facemask for 20 frames ( 7min). We monitored hemoglobin concentrations continuously before, during, and after maternal hyperoxia. Fig. 3 (a) and (b) present a case example of $\mathrm{StO}_{2}$ and $\left[\mathrm{HbO}_{2}\right]$ variation. Overall $(\mathrm{n}=24)$, the method easily resolved changes in placental blood oxygenation due to maternal hyperoxia. $\mathrm{StO}_{2}$ and $\left[\mathrm{HbO}_{2}\right]$ were found to increase, by a median (IQR) of $7.2(6.0,9.0) \%$ and $2.0(1.2,3.4) \mu M$, respectively. 
(a) Placental $\mathrm{StO}_{2}$ change during maternal hyperoxia - exemplary case

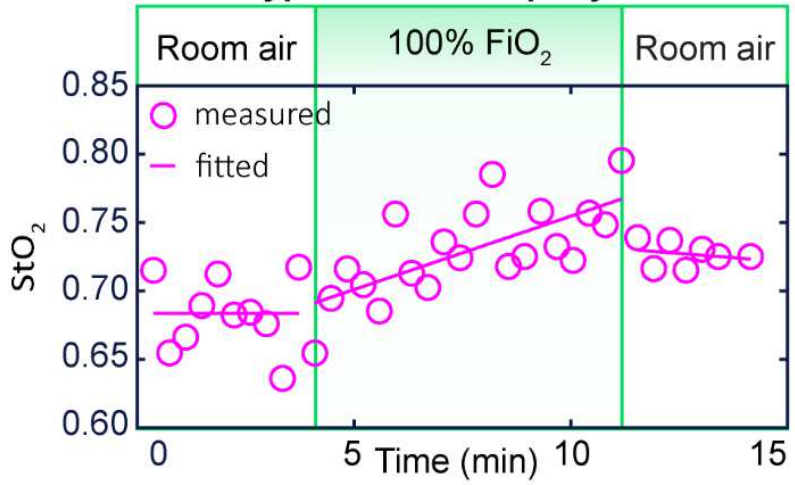

(c) Averaged placental hemodynamic responses to maternal hyperoxia of NPO group $(n=15)$

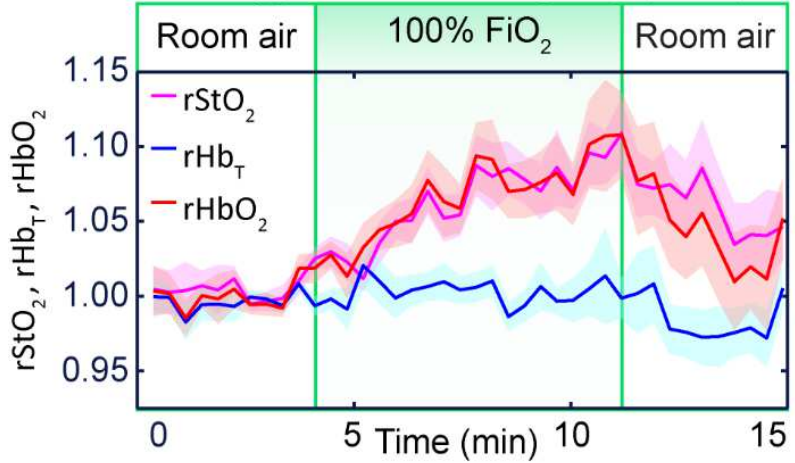

(e) Averaged placental hemodynamic responses to maternal hyperoxia of NPP group $(n=16)$

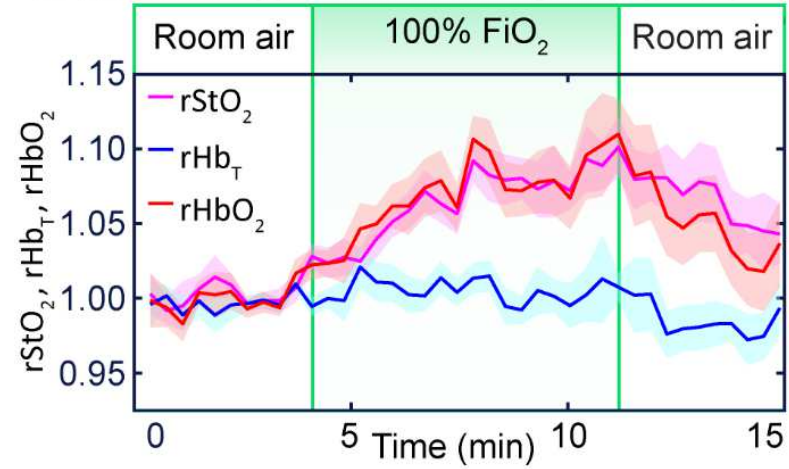

(b) Placental $\mathrm{HbO}_{2}$ change during maternal hyperoxia - exemplary case

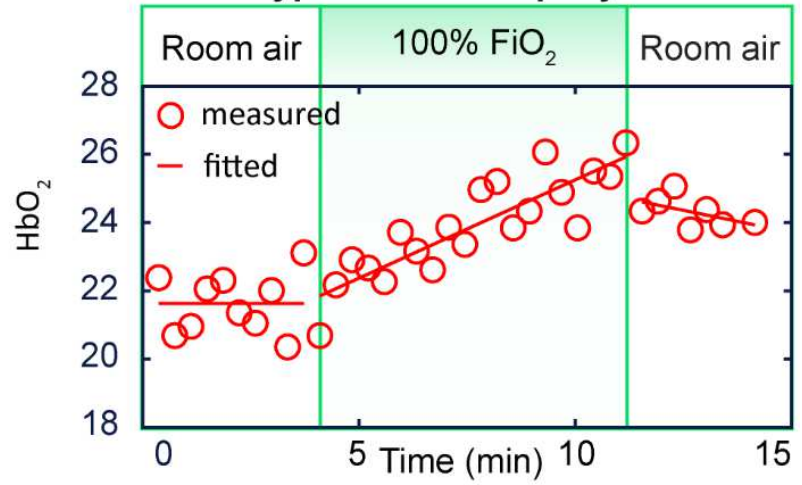

(d) Averaged placental hemodynamic responses to maternal hyperoxia of APO group $(n=9)$

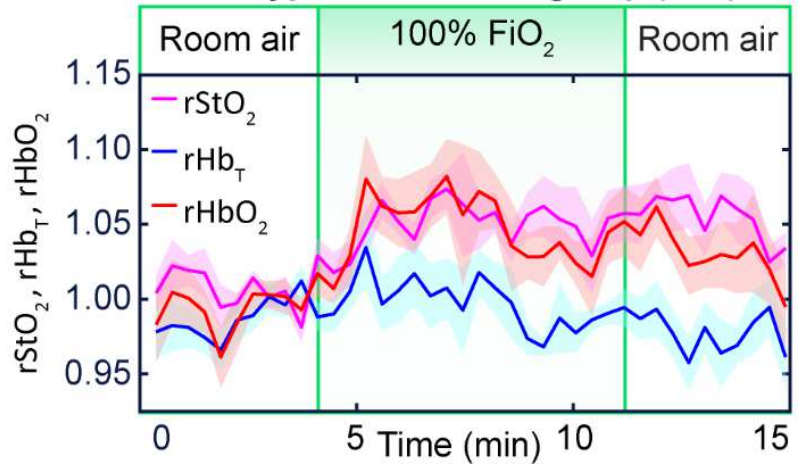

(f) Averaged placental hemodynamic responses to maternal hyperoxia of MVM group $(n=8)$

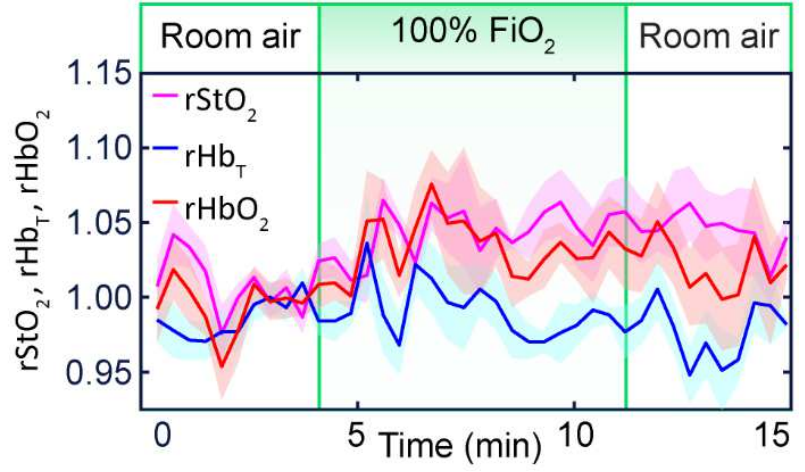

Fig. 3: Continuous monitoring of placental hemoglobin properties during maternal hyperoxia. (a), (b): Exemplary case example of $\mathrm{StO}_{2}$ and $\left[\mathrm{HbO}_{2}\right]$ during maternal hyperoxia. (c), (d): Averaged placental $r S t \mathrm{O}_{2}$ (purple), $r \mathrm{Hb}_{T}$ (blue), and $\mathrm{rHbO}_{2}$ (red) for normal pregnancy outcome (NPO) subjects and for subjects with adverse pregnancy outcome (APO). (e), (f): Averaged placental $r S t O_{2}$ (purple), $r H b_{T}$ (blue), $r \mathrm{HbO}_{2}$ (red) for normal placental pathology (NPP) subjects and for subjects with Maternal Vascular Malperfusion (MVM). Shaded regions represent standard error. Notice, the cohort averaged $r \mathrm{StO}_{2}, \mathrm{rHbO}_{2}$ exhibit a significant increase during maternal hyperoxia for NPO and NPP groups, but a blunted response for APO and MVM groups. 
Optical biomarkers for adverse pregnancy outcome (APO) and maternal vascular malperfusion (MVM).

Another goal of the maternal hyperoxia study was to examine associations between placental oxygen dynamics and both APO and MVM. To this end, APO was defined as a composite of gestational hypertension, preeclampsia, or intrauterine growth restriction, and MVM was determined from examination of delivered placentas by a single placental pathologist (R.L.L.). APO was found in 9 of 24 subjects; MVM was found in 8 of 24 subjects. Note, 2 of the 15 subjects with normal pregnancy outcome (NPO) had MVM, and 3 of the 9 subjects with APO had normal placental pathology (NPP).

Absolute values of $\mathrm{StO}_{2},\left[\mathrm{Hb}_{\mathrm{T}}\right]$, and $\left[\mathrm{HbO}_{2}\right]$ were determined, as well as their variation relative to baseline during maternal hyperoxia, i.e., $r \mathrm{StO}_{2}, r \mathrm{Hb}_{T}$, and $r \mathrm{HbO}_{2}$. Relative data were obtained by normalizing their time series values to the mean of the last four frames of the baseline period.

The 24 subjects were categorized into two groups according to their clinical pregnancy outcomes: NPO or APO. Both $r \mathrm{StO}_{2}$ and $\mathrm{rHbO}_{2}$ increased substantially in response to maternal hyperoxia for the NPO group (Fig. 3 (c)). However, the same parameters in patients with APO showed a more blunted response (Fig. 3 (d)).

Per patient placental histopathology, the same 24 subjects were categorized into two groups: NPP or MVM. We observed significant, large and positive, $\mathrm{rStO}_{2}$ and $\mathrm{rbbO}_{2}$ in the NPP group (Fig. 3 (e)), but these same parameters showed a blunted response in the MVM group (Fig. 3 (f)). $r H b_{T}$ was comparatively constant for all groups.

We next sought to quantitatively determine whether placental hemoglobin properties were significantly associated with APO. For this analysis, mean baseline $\mathrm{StO}_{2},\left[\mathrm{Hb}_{\mathrm{T}}\right]$, and $\left[\mathrm{HbO}_{2}\right]$ were calculated using the final 4 frames of the baseline period. Hyperoxia-induced changes from baseline, i.e., $\Delta \mathrm{StO}_{2}, \Delta \mathrm{Hb}_{\mathrm{T}}$, and $\Delta \mathrm{HbO}_{2}$, were defined using mean values in the 4-frame window wherein maximum $\mathrm{StO}_{2}$ occurred. Baseline $\mathrm{StO}_{2},\left[\mathrm{Hb}_{\mathrm{T}}\right]$, and $\left[\mathrm{HbO}_{2}\right]$ were not associated with APO (Fig. 4(a), Table 1 (a)). On the other hand $\mathrm{HbO}_{2}$ was significantly reduced in cases with APO compared to NPO, and $\mathrm{StO}_{2}$ was also reduced (marginally significant) (Fig. 4(b), Table 1 (a)).

Similarly, we determined whether placental hemoglobin properties were significantly associated with MVM. Baseline $\mathrm{StO}_{2},\left[\mathrm{Hb}_{\mathrm{T}}\right]$, and $\left[\mathrm{HbO}_{2}\right]$ were not associated with MVM (Fig. 5(a), Table 1 (b)). $\Delta \mathrm{HbO}_{2}$ was significantly reduced in cases with $\mathrm{MVM}$, and $\mathrm{StO}_{2}$ was also reduced (marginally significant) (Fig. 5(b), Table 1 (b)). 
(A) Placental hemoglobin properties during baseline for NPO and APO pregnancies
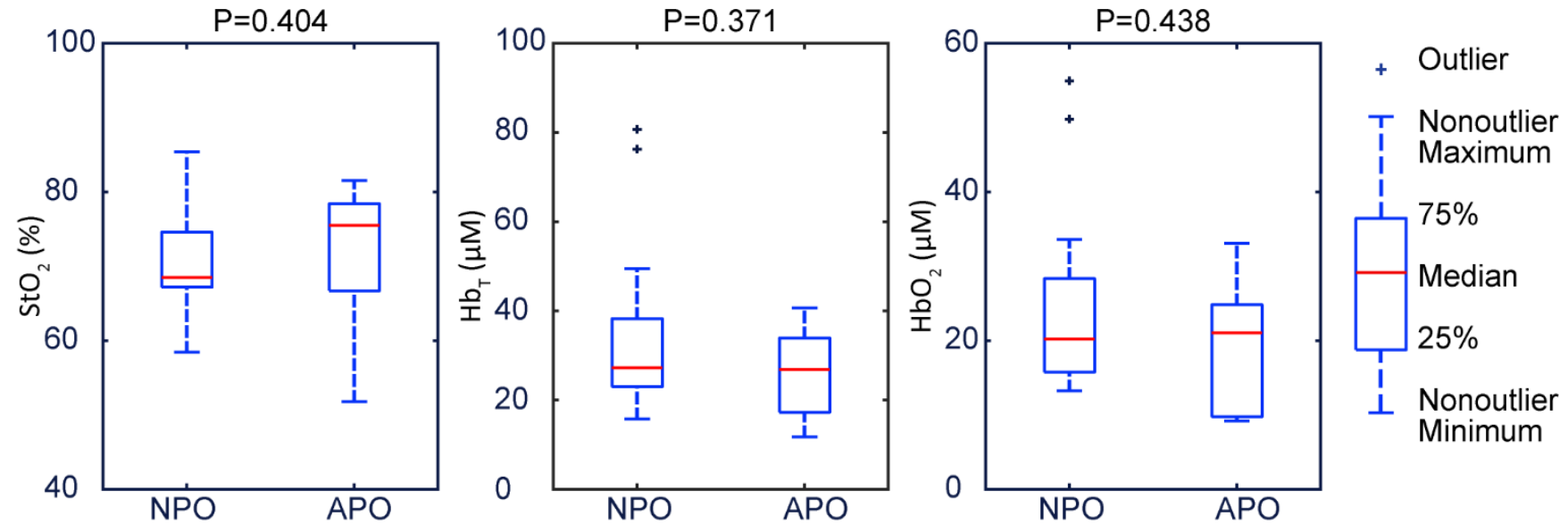

(B) Placental hemoglobin changes during maternal hyperoxia for NPO and APO pregnancies
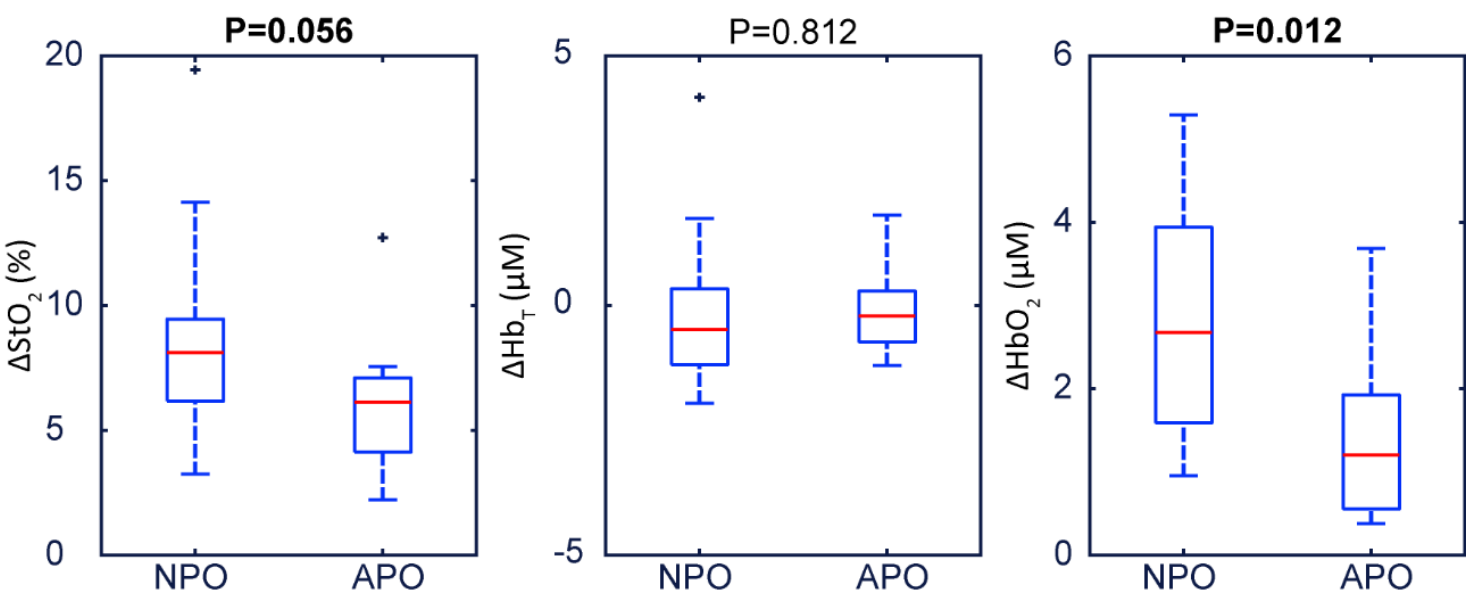

Fig. 4: Boxplots of static and dynamic placental hemoglobin properties during maternal hyperoxia for subjects with normal pregnancy outcome (NPO) $(n=15)$ and Adverse Pregnancy Outcome (APO) (n=9). (a)

Baseline $\mathrm{StO}_{2},\left[\mathrm{Hb}_{\mathrm{T}}\right],\left[\mathrm{HbO}_{2}\right]$ are not associated with pregnancy outcome. (b) $\Delta \mathrm{StO}_{2}, \Delta \mathrm{Hb}_{\mathrm{T}}, \Delta \mathrm{HbO}_{2}$ during maternal hyperoxia. $\Delta \mathrm{StO}_{2}$ and $\Delta \mathrm{HbO}_{2}$ exhibit clear separation amongst subjects with NPO versus APO. P values are calculated by Wilcoxon rank sum test.

8 Our new methodology permitted study of the hemodynamic properties of the adipose and rectus/uterus layer

(see Extended Data Table 4). These adipose and rectus/uterus layer properties, reported for the first time,

typically differed from those of the placenta. Collectively, these data suggest that without multi-layer modeling, the computed placenta responses would have been attenuated, since, without multi-layer modeling, the deep tissue signal would represent a weighted average of placenta, rectus/uterus and adipose layers. 
(a) Placental hemoglobin properties during baseline for NPP and MVM pregnancies
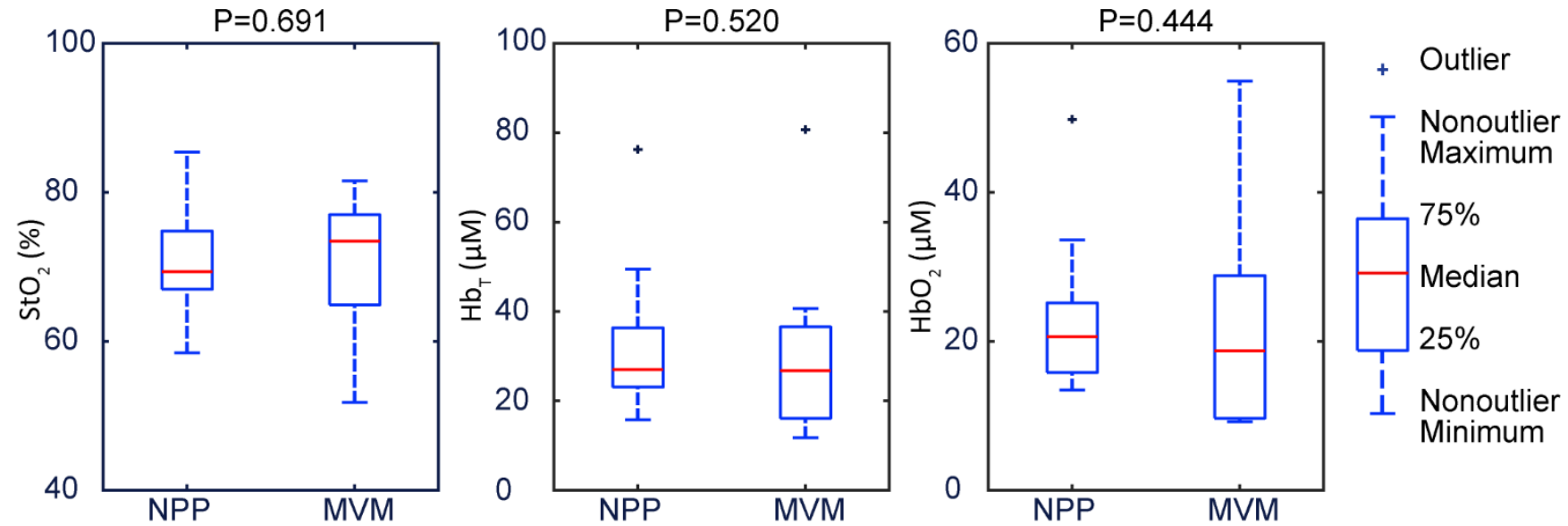

(b) Placental hemoglobin changes during maternal hyperoxia for NPP and MVM pregnancies
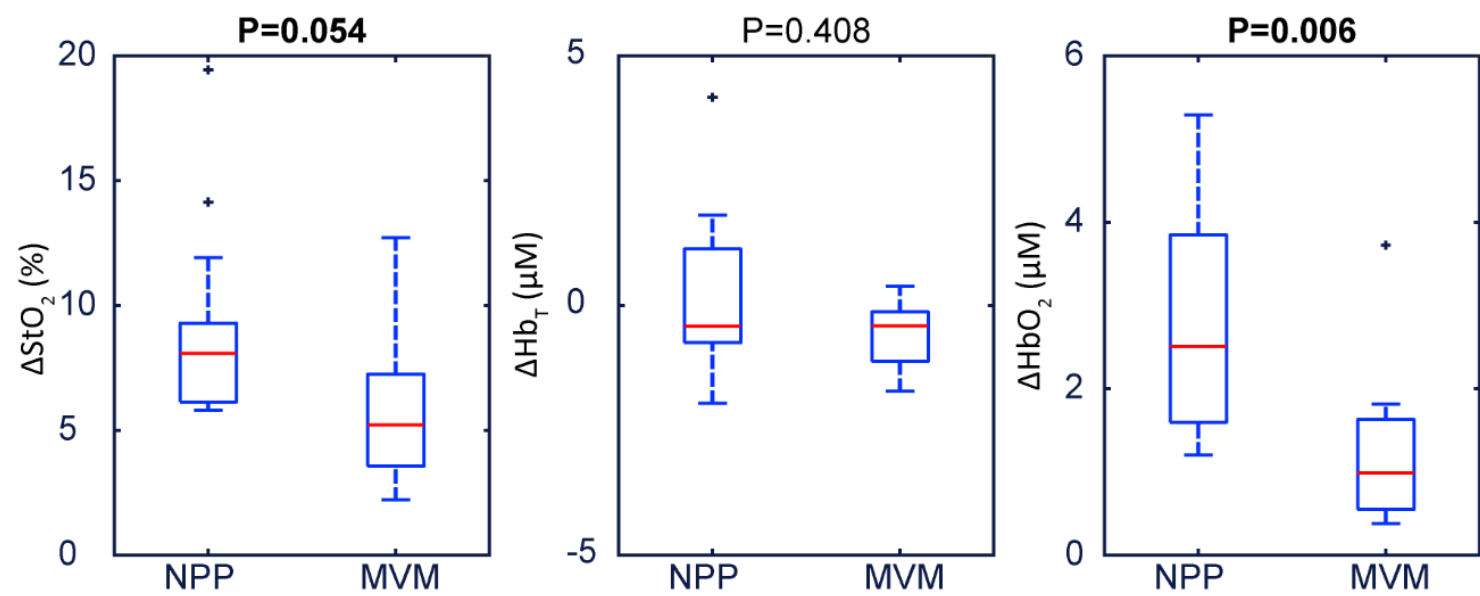

Fig. 5: Boxplots of static and dynamic placental hemoglobin properties during maternal hyperoxia for subjects with normal placental pathology (NPP) $(n=16)$ and Maternal Vascular Malperfusion (MVM) $(n=8)$.

(a) Baseline $\mathrm{StO}_{2},\left[\mathrm{Hb}_{\mathrm{T}}\right],\left[\mathrm{HbO}_{2}\right]$ are not associated with placental pathology. (b) $\Delta \mathrm{StO}_{2}, \Delta H b_{T}, \Delta \mathrm{HbO}_{2}$ during maternal hyperoxia. $\Delta \mathrm{StO}_{2}$ and $\Delta \mathrm{HbO}_{2}$ exhibit clear separation amongst subjects with NPP versus MVM. P values are calculated by Wilcoxon rank sum test.

We also carried out the same univariate analysis with more standard parameters such as maternal age,

9 nulliparity, gestational age (GA) at study visit, maternal BMI, placental depth $(d)$, and uterine artery Doppler

10 pulsatility index (UtA PI) which has been proposed as surrogate indicator of trophoblastic invasion ${ }^{39}$. We did not

11 find significant association between APO and/or MVM and either maternal age, nulliparity, GA at study visit, or

12 UtA PI (Table 1 (c) (d)). However, we did observe a trend towards larger $d$ and BMI in subjects with APO 
$(\mathrm{P}=0.065, \mathrm{P}=0.095$, respectively). Thus, although our sample size is small and although these variables are not necessarily uncorrelated, for completeness we ran binary logistic regressions with pairs of variables: $d, \Delta \mathrm{StO}_{2} ; d$, $\Delta \mathrm{HbO}_{2}$; $\mathrm{BMI}, \Delta \mathrm{StO}_{2}$; $\mathrm{BMI}, \Delta \mathrm{HbO}_{2}$. The results confirmed that there remained a trend towards a significant

4

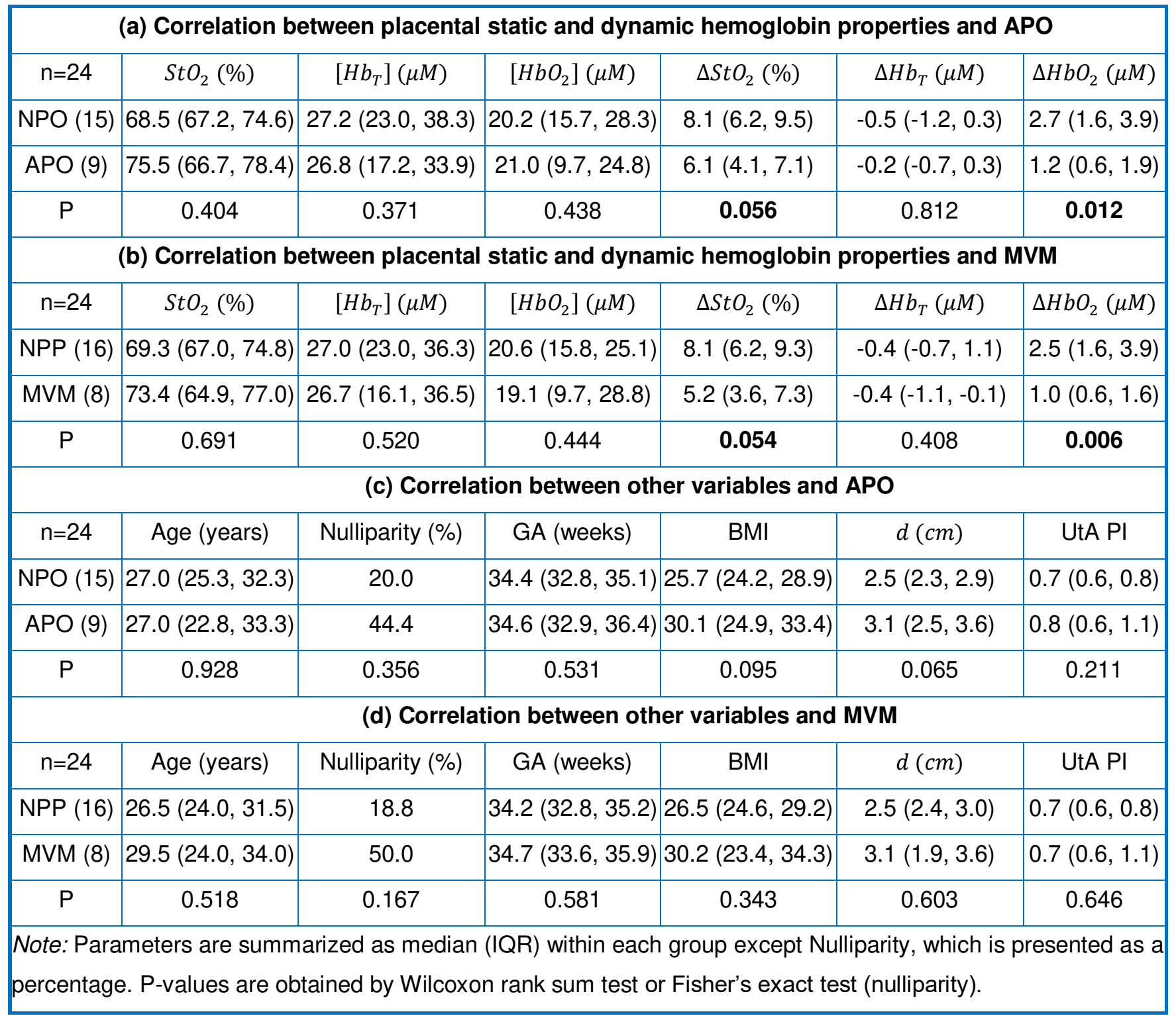

Table 1: Correlation of hemodynamic and demographic variables with placental dysfunction

\section{(a) Correlation between placental static and dynamic hemoglobin properties and APO}




\section{Discussion}

Direct and non-invasive methods to assess placental function in vivo and at the bedside do not currently exist. The depth of the placenta below the skin surface, and the variability in the properties of the overlying layers, presents significant challenges for optical diagnostics. Here we developed and reported on the performance of a novel instrument and methodology that dramatically expands the capabilities of DOS to enable real-time, continuous, dynamic monitoring of organs buried far below the tissue surface, such as the human placenta.

We demonstrated the instrument has sufficient dynamic range and SNR to perform measurements at long SDSs (up to $10 \mathrm{~cm}$ ) so that deeper layers can be optically interrogated. Moreover, by coupling FD-DOS instrumentation with US imaging, we directly map the morphology of overlying layers of the abdominal wall and uterus. This mapping permits multi-layer modeling of tissue properties that effectively isolates placenta optical/physiological properties. We validated the methodology using tissue phantoms and finite element simulations, and we carried out a pilot study of $3^{\text {rd }}$ trimester pregnancies.

Placental $\mathrm{StO}_{2},\left[\mathrm{Hb}_{\mathrm{T}}\right]$, and $\left[\mathrm{HbO}_{2}\right]$ were noninvasively measured at the bedside for the first time, and the repeatability/stability of the optical metrics were demonstrated. Since gold standard references for placental tissue hemoglobin values do not exist, we used functional perturbations to show sensitivity to expected physiologic changes. Maternal left lateral tilt positioning relieves caval compression and augments venous return to the heart, improving cardiac output. In pregnancy, $20 \%$ of maternal cardiac output is directed to uteroplacental perfusion. Thus, this maneuver generally increases blood flow to the placenta. In fact, this position is used clinically as a fetal resuscitation maneuver during labor to maximize placental perfusion ${ }^{40}$. Despite the limited sample size $(n=3)$, the FD-DOS/US device detected an increase for both $\left[\mathrm{Hb}_{\mathrm{T}}\right]$ and $\left[\mathrm{HbO}_{2}\right]$, consistent with the expected increased maternal blood flow into the intervillous vascular spaces of the placenta.

More importantly, the maternal hyperoxia experiments demonstrated that the method can detect real time changes in placental $\left[\mathrm{HbO}_{2}\right]$ and $\mathrm{StO}_{2}$ due to inflow of the oxygen-enriched maternal blood into the placenta, while the $\left[H b_{T}\right]$ remained comparably stable. These perturbation experiments validated clinical expectation, and confirmed sensitivity of these novel optical measures to underlying utero-placental hemodynamics.

Finally, we studied how optical metrics obtained during maternal hyperoxia correlate with pregnancy outcome and placental pathology. Adverse pregnancy outcomes (APOs) are significantly associated with both short- and 
1 long-term morbidity and mortality ${ }^{1-3}$. Similarly, MVM are associated with both APOs and long-term adverse outcomes $^{33}$, with prevalence estimates as high as $32 \%$ in term births and over $50 \%$ in preterm births ${ }^{41}$. Our pilot study indicates that non-invasive, continuous optical monitoring of placental response to maternal hyperoxia is feasible and holds potential as a methodology to detect signs of poor placental perfusion weeks before delivery.

The need for new tools to assess placental function is well-known to clinicians, and as a result, critical knowledge gaps exists in obstetrical care. For this reason, some investigators have turned to MRI to derive functional parameters related to placental oxygenation. MRI does not have the same issues related to depth of penetration that limit optics, and specialized MRI techniques such as $\mathrm{T}_{2}{ }^{*}, \mathrm{BOLD}$, and MR susceptibility are correlated with oxygen content ${ }^{10-12}$. However, these correlations with oxygen have limitations, and MRI is not suitable for bedside measurements. DOS, by contrast, can directly measure deoxy- and oxy-hemoglobin concentrations continuously and non-invasively at the bedside. Thus, although the FD-DOS/US methodology is restricted to probing anterior placentas within $4-5 \mathrm{~cm}$ of the surface, and although the placenta optical signals are due to a combination of maternal and fetal blood, the methodology has clinical promise. Here we have demonstrated a first instrument to permit quantitative dynamic monitoring of human placenta at the bedside, and the associations we have found with APO and MVM support its continued development. However, a larger sample size is desirable to corroborate these findings, and to permit more sophisticated statistical analyses that explore the effects of possible confounding variables and that generate composite metrics with improved specificity and sensitivity.

Further refinement of this technology is important. With improved spatial information from ultrasound, such as 3D imaging, we can improve on the uniform slab-layer tissue model and thereby derive optical properties with greater fidelity. With improved time-resolution, temporal response to functional activation could be explored as a test variable; this would increase sensitivity to changing conditions and facilitate efficient evaluation of multiple placental sites, which is especially important given the potential heterogeneity of pathology within the placenta. In addition, exploration of placental oxygenation at earlier gestational ages, though challenging, could lead to identification of early signs of placental insufficiency. Broadly, we anticipate that optical metrics of placental hemodynamics will enable scientists to understand placental pathophysiology better. Finally, the new instrumentation and methodology is well suited for in vivo study of oxygen function in other internal organs buried deep below the tissue surface, such as the uterus and kidney. 


\section{Methods}

Deep penetration-depth FD-DOS Instrumentation. The details of the construction and operation of the custom heterodyne FD-DOS instrument are schematically shown in Extended Data Fig. 1. Briefly, three sinusoidal electromagnetic waves at radio frequency $(\mathrm{RF})\left(f_{1}=100 \mathrm{MHz}\right)$, i.e., one for each laser diode, and one sinusoidal wave at frequency $\left(f_{2}=100.2 \mathrm{MHz}\right)$, were generated from four low-noise, fractional- $\mathrm{N}$ phase-locked-loop signal generators (HMC833, Hittite Microwave Corporation) and were synchronized by an ultra-low-jitter programmable reference clock ( $f_{0}=50 \mathrm{MHz}$, LMK61E2, Texas Instruments). Each $f_{1}$ wave from the signal generator was amplified (ZX60-P103LN+ by Mini-Circuits), filtered (DC to $98 \mathrm{MHz}$, SLP-100+, Mini-Circuits), and divided (2-way splitter, Z99SC-62-S+, Mini-Circuits) into two $f_{1}$ waves; one was prepared for the reference signal ('reference $f_{1}$ ') and the other was prepared for driving amplitude modulation for one laser ('signal $f_{1}$ '). Simultaneously, the $f_{2}$ wave from the signal generator was also amplified, filtered, and divided (4-way splitter, ZB4PD-52-20W-S+, MiniCircuits) into four $f_{2}$ waves, three were prepared for frequency-mixing with the three detected signals ('signal $f_{2}$ ') and the other was prepared for frequency-mixing with the reference signal ('reference $f_{2}$ ').

The three of 'signal $f_{1}$ ' were further amplified and input into laser controllers (CLD1011LP, Thorlabs), which drive the light amplitude modulation of the three near-infrared lasers with wavelengths of $785 \mathrm{~nm}$ (LP785-SF1000, Thorlabs), 808 nm (LDPC-T3-808-62.5/125-M-35-3S-3-0.5-70-150LD, OZ Optics), and 830 nm (LDPC-T3-83062.5/125-M-35-3S-3-0.5-70-150LD, OZ Optics). The laser controllers also maintain thermal stability using digital proportional-integral-derivative control. To enhance signal-to-noise ratio (SNR), the achievement of a modulation depth (amplitude modulation index), i.e., the ratio of the modulation excursions of the RF signal to the level of unmodulated carrier, of greater than $90 \%$ for each laser is critically important. Achievement of these large modulation depths required individually optimized RF amplification according to each laser's characteristics. Specifically, each 'signal $f_{1}$ ' wave was divided into four sub-signals via a 4-way power splitter. Each sub-signal was then amplified in one or two amplification stages (in each stage, the signal was amplified by $\sim 8.6 \mathrm{~dB}$ ); the 830nm channel had 2 amplification stages and the $785 \mathrm{~nm}$ and $805 \mathrm{~nm}$ channels had 1 amplification stage. The sub-signals were then combined via a 4-way power splitter. A custom-built circuit containing a low noise amplifier (ZX60-P103LN+, Mini-Circuits) and low pass filter (DC to 98 MHz, SLP-100+, Mini-Circuits) was used in each amplification stage. 
The three amplitude modulated laser diodes were fiber-coupled to an optical switch (MEMS 91545C4, Dicon), which was in turn connected to the 10 source fibers (400 $\mu \mathrm{m}$ core, $0.5 \mathrm{NA}$, FP400URT-Custom, Thorlabs) on the probe head (see main text Fig. 1 (b)). The optical switch sequentially cycled each laser diode through each source position and also a "dark count position" (i.e., a cycle of $3 \times 11=33$ sequential measurements; 21 seconds per cycle). Of note, for the dark count measurement, no fiber was connected to the $11^{\text {th }}$ position on the switch (i.e., no light was delivered to the tissue).

Multiply scattered light emerging from the tissue at the detector position was collected by a high-transmission liquid light guide ( $5 \mathrm{~mm}$ core, $0.59 \mathrm{NA}$, LLG5-8H, Thorlabs) that was coupled to a high-sensitivity photomultiplier (PMT) detector (R12829, Hamamatsu). The PMT converts the diffuse light wave to a proportional electric voltage signal, which is then amplified and filtered by a high-speed current amplifier (DHPCA-100, FEMTO), and finally frequency-mixed (ZP-3-S+, Mini-Circuits) with one 'signal $f_{2}$ '. Mixing produces a heterodyne down-converted signal, related to the diffusive light wave (i.e., its amplitude and phase are proportional to those of the diffusive light wave), at frequency $\Delta f=f_{1}-f_{2}=0.2 \mathrm{MHz}$. This lower-frequency signal, which can be very accurately quantified, is the heterodyne detected signal. Simultaneously, a reference signal with the fixed frequency $\Delta f$, amplitude $\left(A_{r}\right)$, and phase $\left(\varphi_{r}\right)$ is generated by mixing the 'reference $f_{1}$ ' and 'reference $f_{2}$ '. A high-sampling-rate lock-in amplifier (MFLI $500 \mathrm{kHz}$, Zurich Instruments) compares reference and detected signals to derive the amplitude $(A)$ and phase $(\varphi)$ of the diffuse light wave (i.e., the lock-in output in-phase $(I=A \cos \varphi)$ and quadrature $(Q=A \sin \varphi)$ signals, from which $A$ and $\varphi$ are calculated). Note, a 3-to-1 RF switch (G4J-520120, Charter Engineering Inc.) was employed to pair the correct reference signal with the corresponding detected wavelength. Note also, prior to computing $A$ and $\varphi$, the $Q$ and $I$ for each wavelength at every source position were subtracted by the corresponding $Q_{\text {noise }}$ and $I_{\text {noise }}$ obtained from the dark count position in the same cycle. In summary, we collect diffuse light waves from 10 source-detector pairs with source-detector separations (SDSs) ranging from $\sim 1$ to $\sim 9 \mathrm{~cm}$ in the patient probe; these data enable the depth-dependent optical determination of tissue properties.

Three-layer photon diffusion model and associated Green's function. The human abdomen is multi-layered. We model it as a three-layer medium wherein each layer is assumed homogeneous and laterally infinite in extension. The experimental geometry is conveniently described using cylindrical coordinates in the main text Fig. 1(c); the depth is denoted by $z(\mathrm{~cm})$ and the source-detector separation (SDS) is denoted by $\rho(\mathrm{cm})$. Both 
source and detector are positioned on the surface $(z=0)$. In the diffusive medium, sources on the tissue boundary are well-modeled as an isotropic point source in the medium at depth of $z_{0}=l_{t r, 1}(\mathrm{~cm})$, which depends on tissue optical properties and is defined below. The diffusion equations (in the frequency-domain) for the

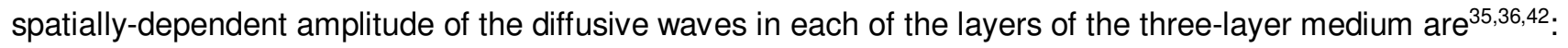

$$
\begin{array}{lr}
\nabla^{2} U_{1}(\rho, z)-\left(\frac{\mu_{a, 1}}{D_{1}}+i \frac{2 \pi f_{1}}{v_{1} D_{1}}\right) U_{1}(\rho, z)=-\frac{M S_{0}}{D_{1}} \delta\left(\rho-\rho_{0}, z-z_{0}\right) ; & 0 \leq z<d_{1} ; \\
\nabla^{2} U_{2}(\rho, z)-\left(\frac{\mu_{a, 2}}{D_{2}}+i \frac{2 \pi f_{1}}{v_{2} D_{2}}\right) U_{2}(\rho, z)=0 ; & d_{1} \leq z<d_{1}+d_{2} ; \\
\nabla^{2} U_{3}(\rho, z)-\left(\frac{\mu_{a, 3}}{D_{3}}+i \frac{2 \pi f_{1}}{v_{3} D_{3}}\right) U_{3}(\rho, z)=0 ; & d_{1}+d_{2} \leq z .
\end{array}
$$

Here, the diffusive wave $\Phi_{A C, k}(\rho, z, t)=U_{k}(\rho, z) e^{i 2 \pi f_{1} t}\left(W_{c m}^{-2}\right)$; it is a complex representation of photon fluence rate within layer $k(k=1,2,3) . S_{0}(W)$ is the time averaged power emitted by the light source, $f_{1}(\mathrm{~Hz})$ and $M$ (dimentsionless) are the frequency $\left(f_{1}=100 \mathrm{MHz}\right)$ and modulation depth of the source, respectively. The source is point-like and located at $\left(\rho_{0}, z_{0}\right) . d_{k}(\mathrm{~cm}), \mu_{a, k}\left(\mathrm{~cm}^{-1}\right)$, and $\mu_{s, k}^{\prime}\left(\mathrm{cm}^{-1}\right)$ are the layer $k$ thickness, layer $k$ light absorption coefficient, and layer $k$ reduced scattering coefficient, respectively. $l_{t r, k} \equiv 1 /\left(\mu_{a, k}+\mu_{s, k}^{\prime}\right)(\mathrm{cm})$ and $D_{k}=(1 / 3) l_{t r, k}(\mathrm{~cm})$ are the photon transport mean-free path and the diffusion coefficient of layer $k \cdot v_{k}=$ $c / n_{k}(\mathrm{~cm} / \mathrm{s})$ is the light velocity in layer $k$, where $c(\mathrm{~cm} / \mathrm{s})$ is the speed of light in vacuum and $n_{k}$ is the refractive index of layer $k$. The boundary conditions for the photon fluence rate and its normal derivative across the interfaces are well-known and are used to derive solutions ${ }^{43}$.

Assuming large SDS, the equations can be solved using a Fourier transform approach and extrapolated-zero boundary conditions. The analytical Green's function for the three-layer diffusion equation in the $z=0$ plane is:

$$
\begin{aligned}
& G_{3 L}\left([\rho, z=0],\left[\rho_{s}=\rho_{0}, z_{s}=z_{0}\right]\right)=\frac{1}{2 \pi} \int_{0}^{\infty} \tilde{G}_{3 L}(s) s J_{0}(s \rho) d s ; \\
& \tilde{G}_{3 L}(s)=\frac{Z_{3 L}(s)}{Y_{3 L}(s)} / \alpha_{1} D_{1} ; \\
& Z_{3 L}(s)=N_{12} M_{23} \exp \left(\alpha_{1} z_{0}-\alpha_{1} d_{1}+\alpha_{2} d_{2}+\alpha_{1} z_{b, 1}\right)-N_{12} M_{23} \exp \left(\alpha_{1} z_{0}-\alpha_{1} d_{1}+\alpha_{2} d_{2}-\alpha_{1} z_{b, 1}\right) \\
& \quad+N_{12} N_{23} \exp \left(-\alpha_{1} z_{0}+\alpha_{1} d_{1}-\alpha_{2} d_{2}+\alpha_{1} z_{b, 1}\right)-N_{12} N_{23} \exp \left(-\alpha_{1} z_{0}+\alpha_{1} d_{1}-\alpha_{2} d_{2}-\alpha_{1} z_{b, 1}\right) \\
& \quad+M_{12} N_{23} \exp \left(\alpha_{1} z_{0}-\alpha_{1} d_{1}-\alpha_{2} d_{2}+\alpha_{1} z_{b, 1}\right)-M_{12} N_{23} \exp \left(\alpha_{1} z_{0}-\alpha_{1} d_{1}-\alpha_{2} d_{2}-\alpha_{1} z_{b, 1}\right) \\
& \quad+M_{12} M_{23} \exp \left(-\alpha_{1} z_{0}+\alpha_{1} d_{1}+\alpha_{2} d_{2}+\alpha_{1} z_{b, 1}\right)-M_{12} M_{23} \exp \left(-\alpha_{1} z_{0}+\alpha_{1} d_{1}+\alpha_{2} d_{2}-\alpha_{1} z_{b, 1}\right) ;(2-3)
\end{aligned}
$$




$$
\begin{aligned}
Y_{3 L}(s) & =M_{12} M_{23} \exp \left(\alpha_{1} d_{1}+\alpha_{2} d_{2}+\alpha_{1} z_{b, 1}\right)+N_{12} M_{23} \exp \left(-\alpha_{1} d_{1}+\alpha_{2} d_{2}-\alpha_{1} z_{b, 1}\right) \\
& +N_{12} N_{23} \exp \left(\alpha_{1} d_{1}-\alpha_{2} d_{2}+\alpha_{1} z_{b, 1}\right)+M_{12} N_{23} \exp \left(-\alpha_{1} d_{1}-\alpha_{2} d_{2}-\alpha_{1} z_{b, 1}\right)
\end{aligned}
$$$$
M_{12}=\alpha_{1} D_{1}+\frac{n_{2}^{2}}{n_{1}^{2}} \alpha_{2} D_{2} ; M_{23}=\alpha_{2} D_{2}+\frac{n_{3}^{2}}{n_{2}^{2}} \alpha_{3} D_{3} ; N_{12}=\alpha_{1} D_{1}-\frac{n_{2}^{2}}{n_{1}^{2}} \alpha_{2} D_{2} ; N_{23}=\alpha_{2} D_{2}-\frac{n_{3}^{2}}{n_{2}^{2}} \alpha_{3} D_{3} ;
$$

$$
\alpha_{k}=\frac{\left(D_{\mathrm{k}} s^{2}+v_{k} \mu_{a, k}+i 2 \pi f_{1}\right)}{D_{\mathrm{k}}} ; \quad k=1,2,3 .
$$

Here, $J_{0}$ is the Bessel function of the first kind and zero-order. $z_{b, 1} \equiv(2 / 3) l_{t r, 1}\left(1+R_{e f f} / 1-R_{e f f}\right)(\mathrm{cm})$ is the extrapolation length, where $R_{\text {eff }}$ is related to the indices of refraction of the media ${ }^{43}$. In practice, we solve the integral in equation (2-1) numerically by applying Gauss-Laguerre quadrature of 5000 points. Note, in order to minimize numerical errors, the hyperbolic functions are expanded and simplified.

Note, the analytical Green's function for the two-layer diffusion equation in the $z=0$ plane can be derived from a special case of the three-layer model, where $\mu_{a, 1}=\mu_{a, 2}, \mu_{s, 1}^{\prime}=\mu_{s, 2}^{\prime}, n_{1}=n_{2}, d_{1}+d_{2}=d$, and $D_{1}=D_{2}, \alpha_{1}=\alpha_{2}$.

$$
\begin{aligned}
& G_{2 L}\left([\rho, z=0],\left[\rho_{s}=\rho_{0}, Z_{s}=z_{0}\right]\right)=\frac{1}{2 \pi} \int_{0}^{\infty} \tilde{G}_{2 L}(s) s J_{0}(s \rho) d s \\
& \tilde{G}_{2 L}(s)=\frac{Z_{2 L}(s)}{Y_{2 L}(s)} / \alpha_{1} D_{1} \\
& Z_{2 L}(s)=\exp \left(-\alpha_{1} z_{0}\right)-\exp \left(-\alpha_{1} z_{0}-2 \alpha_{1} z_{b, 1}\right)+\beta \cdot \exp \left(\alpha_{1} z_{0}-2 \alpha_{1} d\right)-\beta \cdot \exp \left(\alpha_{1} z_{0}-2 \alpha_{1} d-2 \alpha_{1} z_{b, 1}\right) ;(3-3) \\
& Y_{2 L}(s)=1+\beta \cdot \exp \left(-2 \alpha_{1} d-2 \alpha_{1} z_{b, 1}\right) ; \\
& \beta=\frac{\alpha_{1} D_{1}-\left(n_{3}^{2} / n_{1}^{2}\right) \alpha_{3} D_{3}}{\alpha_{1} D_{1}+\left(n_{3}^{2} / n_{1}^{2}\right) \alpha_{3} D_{3}} .
\end{aligned}
$$

The photon fluence rate measured on the tissue surface is essentially the Green's function multiplied by the constant amplitude of the source $M S_{0}$. The detected signal intensity is directly proportional this photon fluence rate:

$$
\begin{aligned}
& U_{1}\left([\rho, z=0],\left[\rho_{s}=\rho_{0}, z_{s}=z_{0}\right]\right)=M S_{0} G\left([\rho, z=0],\left[\rho_{s}=\rho_{0}, z_{s}=z_{0}\right]\right) ; \\
& I_{m}(\rho)=K U_{1}\left([\rho, z=0],\left[\rho_{s}=\rho_{0}, z_{s}=z_{0}\right]\right)=C G\left([\rho, z=0],\left[\rho_{s}=\rho_{0}, z_{s}=z_{0}\right]\right) ; \\
& A_{m}(\rho)=\left|I_{m}(\rho)\right| ; \quad \theta_{m}(\rho)=\arg \left[I_{m}(\rho)\right] .
\end{aligned}
$$

Here, $C=C_{a} \exp \left(-i C_{p}\right)$ is the complex light coupling coefficient (a proportionality constant); $C_{a}$ and $C_{p}$, are determined using calibration phantoms with known optical properties before/after the measurement. With 
equations (2) and (4), the signal at the tissue surface boundary can be calculated, given input optical properties for each layer and layer thicknesses. Such a calculation is called a solution of the forward problem. The reconstruction algorithms underlying the FD-DOS analysis solves the inverse problem by finding the tissue properties that minimize the difference between measured signal and the forward problem theoretical solution (with specific input properties).

Global optimization with multi-spectral and multi-SDS reconstruction. In practice, the two-layer or threelayer optical properties based on the photon diffusion model were reconstructed by solving a global optimization problem. Specifically, we carried out the data inversion using all source-detector pairs and wavelengths simultaneously. This approach builds-in global constraints about chromophore absorption and layer geometry into the inverse problem and is critical for robust fitting.

The multispectral fitting assumed that $\mu_{a, k}\left(\lambda_{j}\right)$, the absorption coefficient at layer $k$ at wavelength $\lambda_{j}(j=$ $\left.1,2,3, \lambda_{j}=785,808,830 \mathrm{~nm}\right)$, is due to the absorption of $\left[\mathrm{HbO}_{2}\right]_{k},[\mathrm{Hb}]_{k},\left[\mathrm{H}_{2} \mathrm{O}\right]_{k}$, and $[\text { lipid }]_{k}$.

$$
\mu_{a, k}\left(\lambda_{j}\right)=\varepsilon_{\mathrm{HbO}_{2}}\left(\lambda_{j}\right)\left[\mathrm{HbO}_{2}\right]_{k}+\varepsilon_{\mathrm{Hb}}\left(\lambda_{j}\right)[\mathrm{Hb}]_{k}+\varepsilon_{\mathrm{H}_{2} \mathrm{O}}\left(\lambda_{j}\right)\left[\mathrm{H}_{2} \mathrm{O}\right]_{k}+\varepsilon_{\text {lipid }}\left(\lambda_{j}\right)[\text { lipid }]_{k}
$$

In equation (5), $\varepsilon_{\mathrm{HbO}_{2}}\left(\lambda_{j}\right), \varepsilon_{\mathrm{Hb}}\left(\lambda_{j}\right), \varepsilon_{\mathrm{H}_{2} \mathrm{O}}$, and $\varepsilon_{\text {lipid }}\left(\lambda_{j}\right)$ are wavelength-dependent extinction coefficients for each chromophore which are known ${ }^{44} \cdot\left[\mathrm{H}_{2} \mathrm{O}\right]_{k}$ and $[\text { lipid }]_{k}$ are the concentration of water and lipid in layer $k$, which are assumed ${ }^{44} .\left[\mathrm{HbO}_{2}\right]_{k}$ and $[\mathrm{Hb}]_{k}$ are the concentration of oxy-and deoxy-hemoglobin in layer $k$; they are determined by solving the inverse problem.

Notice, the total hemoglobin concentration $\left[\mathrm{Hb}_{\mathrm{T}}\right]_{k}$ and tissue blood oxygen saturation $\mathrm{StO}_{2, k}$ in layer $k$ can also be readily obtained from:

$$
\left[\mathrm{Hb}_{\mathrm{T}}\right]_{k}=\left[\mathrm{HbO}_{2}\right]_{k}+[\mathrm{Hb}]_{k} ; \quad S t \mathrm{O}_{2, k}=\left[\mathrm{HbO}_{2}\right]_{k} /\left[\mathrm{Hb}_{\mathrm{T}}\right]_{k} ; \quad k=1,2,3 .
$$

For multi-spectral fitting, we also assumed a Mie scattering model (below) for the tissue scatterers ${ }^{15}$, wherein the scattering coefficient in layer $k$ is a power law function with scattering amplitude $\gamma_{k}$ and scattering power $b_{k}$. Here, $\lambda_{0}=700 \mathrm{~nm}$ is a reference wavelength chosen based on the range of the three wavelengths.

$$
\mu_{s, k}^{\prime}\left(\lambda_{j}\right)=\gamma_{k}\left(\lambda_{j} / \lambda_{0}\right)^{-b_{k}}
$$

With equations (5), (6), and (7), StO $\mathrm{Z}_{2, k}$ and $\left[\mathrm{Hb}_{\mathrm{T}}\right]_{k}$ can be directly determined by a global optimization using all the data: 


$$
\operatorname{argmin} \sum_{j=1}^{3} \sum_{l=1}^{L}\left|\varepsilon_{l, j}(\bar{X})\right|^{2}
$$

$$
\Psi(\bar{X})=\left|\varepsilon_{l, j}(\bar{X})\right|^{2}=\sum_{j=1}^{3} \sum_{l=1}^{L}\left\|\ln \left[A_{c, l, j} / A_{c, l_{0}, j}\right]-\ln \left[A_{m, l, j} / A_{m, l_{0}, j}\right]\right\|^{2}+\left\|\left[\theta_{c, l, j}-\theta_{c, l_{0}, j}\right]-\left[\theta_{m, l, j}-\theta_{m, l_{0}, j}\right]\right\|^{2} ;(8-2)
$$

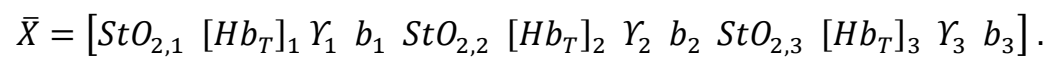

In this global optimization, $\bar{X}$ is an array of all fitting variables, including hemoglobin concentrations and variants thereof and Mie scattering model parameters for scattering. The objective function $\Psi(\bar{X})$ is a "residual" function of $\bar{X}$; it is essentially a Chi-squared function that compares calculated to measured data. $\|\quad\|^{2}$ represents the $L^{2}$ norm of the vector. $A_{c, l, j}$ and $\theta_{c, l, j}$ are the calculated (predicted) amplitude and phase using the forward solver with estimated optical properties. $A_{m, l, j}$ and $\theta_{m, l, j}$ are the measured amplitude and phase. Subscripts $l$ and $j$ represent the $l-t h$ SDS and $j-t h$ wavelength, respectively. Notice, the normalization amplitude and phase factors are also incorporated into the objective function; these terms are denoted with the subscript, $l_{0}$.

Regularization and initialization of optimization problem. To avoid overfitting in the reconstruction, regularization is employed to reduce the ill-posedness of the inverse problem. A Tikhonov regularization term is added to the original objective function to provide additional constraints. Thus, the new objective function $\Psi^{\prime}(\bar{X})$ is the sum of the residual function in (8-2) and a weighted regularization term ${ }^{45}$ :

$$
\Psi^{\prime}(\bar{X})=\sum_{j=1}^{3} \sum_{l=1}^{L}\left|\varepsilon_{l, j}(\bar{X})\right|^{2}+\zeta_{R} R(\bar{X}) ; \quad R(\bar{X})=\left\|\bar{X}-\bar{X}^{(0)}\right\|^{2}
$$

$R(\bar{X})$ is the Tikhonov regularization term, and $\zeta_{R}$ is the regularization hyperparameter, which calibrates the relative weight of the residual function and regularization term. Tikhonov regularization, as used here, seeks to minimize the difference between the initial estimated value $\bar{X}^{(0)}$ and the reconstructed value $\bar{X}$. The value for the regularization hyperparameter is determined using an L-curve method, which is a convenient graphical tool to find the optimized regularization parameter which balances the trade-off between fluctuation size and fluctuation smoothing. Once the $\zeta_{R}$ is determined, the optimization problem to minimize $\Psi^{\prime}(\bar{X})$ can be solved iteratively. In practice, the iterative algorithm is performed by MATLAB fmincon function with a parallel search function- multistart for global minimum ${ }^{46}$. 
$1 \bar{X}^{(0)}$ is set. It is important that the initial guess is chosen to be reasonably close to the true value; otherwise the iterative search may not converge to a meaningful solution. Main text Fig. 2 (a) schematically outlines our threestep reconstruction procedure for initialization and determination of placental hemodynamic properties.

In essence, each step of the three-step reconstruction finds "best" tissue properties by minimizing the difference between measured data and the predictions of diffuse optical tissue models of increasing complexity. The STEP 1 assumes the underlying tissue is semi-infinite and homogeneous; it utilizes short SDSs to derive an initial estimate for properties of the near-surface region and the full set of SDSs to derive an initial property estimate for the whole region. For predictions, a standard semi-infinite homogeneous medium analytic solution is used in step one.

The STEP 2 utilizes estimates from step one as initial guesses in an analytic two-layer diffuse optical tissue model. The two-layer (and three-layer) models fix layer thicknesses based on US data, but other tissue properties within each layer such as scattering, $\mathrm{StO}_{2}$, and $\left[\mathrm{Hb}_{\mathrm{T}}\right]$, are permitted to vary to minimize the difference between measurement and tissue model predictions. Short SDSs and a two-layer analytical light transport model are utilized to derive best estimates for properties of adipose and rectus/uterus layers; all SDSs and this same twolayer analytic light transport model are utilized to derive best estimates for the over-layer (adipose plus rectus/uterus) and placental regions.

The STEP 3 utilizes estimates for each layer from step two as initial guesses in the final three-layer diffuse optical tissue model. Again, all layer thicknesses are fixed by ultrasound image segmentation, but the other tissue properties within each layer are permitted to vary to minimize the difference between the measurement and the predictions of the three-layer analytic light transport model.

\section{Experimental validation/characterization with tissue-simulating phantoms. We characterized FD-DOS} instrument performance using tissue-simulating phantoms. The simplest tissue phantoms were comprised of water, ink for absorption, and $20 \%$ Intralipid (Baxter) for scattering. Briefly, the detector fiber was fixed at the liquid surface and a source fiber was physically translated in the same surface plane with SDSs ranging from $6.2 \mathrm{~cm}$ to $10 \mathrm{~cm}$ using a translation stage (see main text Fig. 2 (b)). To good approximation, the coupling coefficients in equation (4) were the same at every SDS, and a nonlinear fitting based on semi-infinite homogeneous solutions of the diffusion equation was employed to reconstruct tissue phantom optical properties ${ }^{13}$. The results 
demonstrate good SNR at SDSs up to $10 \mathrm{~cm}$ (see main text Fig. 2 (b)) and accuracies in the range of 3\% to $9 \%$.

In the two-layer phantom experiments, a solid phantom $\left(\mu_{a, b}=0.10 \mathrm{~cm}^{-1}, \mu_{s, b}^{\prime}=5.00 \mathrm{~cm}^{-1}\right.$ for $\left.\lambda_{1}=785 \mathrm{~nm}\right)$ was positioned under the liquid phantom, and the optical probe was set on the liquid surface (see main text Fig. 2 (c)). An absorption-titration experiment tested sensitivity to absorption coefficient (chromophore concentration). The over-layer liquid phantom thickness was held constant at $3 \mathrm{~cm}$, the over-layer scattering coefficient was held constant $\left(\mu_{s, t}^{\prime}=9.10 \mathrm{~cm}^{-1}\right.$ for $\left.\lambda_{1}=785 \mathrm{~nm}\right)$, and the absorption coefficient was incrementally increased in the top layer from $\mu_{a, t}=0.08$ to $0.13 \mathrm{~cm}^{-1}$. Results are given in Extended Data Table 1 (left). A depth-changing experiment tested sensitivity to superficial layer thickness (see main text Fig. 2 (c)). Here, the liquid had fixed optical properties $\left(\mu_{a, t}=0.13 \mathrm{~cm}^{-1}, \mu_{s, t}^{\prime}=9.10 \mathrm{~cm}^{-1}\right.$ for $\left.\lambda_{1}=785 \mathrm{~nm}\right)$, and the superficial layer thickness was increased from 1.5 to $3.0 \mathrm{~cm}$. Results are given in Extended Data Table 1 (right). In total, the experimental results demonstrated the instrument can extract deep layer tissue optical properties accurately, with errors less than $15 \%$ (absorption coefficients with error less than 10\%).

Ultrasound image segmentation. The US transducer at the center of optical probe was critical because it enabled us to derive tissue layer morphology and geometry information needed for the optical reconstruction algorithms. At the beginning of each 'optical frame', we captured an US image and measured the depth (below the tissue surface) of adipose, rectus, uterus, and placenta tissue layers in the left, middle and right sections of the US image. The difference between the left, middle and right depths was thus determined to ensure effective reconstruction of tissue optical properties. Note, if this difference was less than $0.5 \mathrm{~cm}$, then we took data; on the rare occasion when the difference was larger than $0.5 \mathrm{~cm}$, we repositioned the probe position/angle to decrease the difference and then took data.

Pregnancy Outcome. After delivery, medical records were reviewed and relevant data on outcomes were extracted by a reviewer blinded to the optical data. Gestational hypertension (GHTN) and preeclampsia (PE) were defined per American College of Obstetricians and Gynecologists criteria ${ }^{47}$; intrauterine growth restriction (IUGR) was defined as a birth weight below the $5^{\text {th }}$ percentile for gestational age ${ }^{48}$.

Placental Histopathology. The delivered placentas were evaluated using a standard procedure ${ }^{34,49}$ by a single placental pathologist (R.L.L.) who was blinded to the optical properties. Maternal vascular malperfusion (MVM) was defined as a pattern of injury including placental hypoplasia (small for gestational age), villous infarcts, 
retroplacental hemorrhage (abruptio placentae), distal villous hypoplasia, villous agglutination, accelerated villous maturation and decidual arteriopathy. The minimum findings required for MVM diagnosis included decidual arteriopathy or at least 2 other features including accelerated villous maturation.

In vivo monitoring of placental oxygen dynamics. We designed a pilot clinical study of human placental oxygen-related hemodynamic properties. The study enrolled women with singleton pregnancies in the third trimester, anterior placentas, and pre-gravid $\mathrm{BMl}<40$. For each experiment, subjects were placed in supine, semirecumbent position, and the central region of placenta was monitored. Prior to proceeding with the study, approval by the Institutional Review Board (IRB) at the University of Pennsylvania was obtained. Each subject signed the resultant informed consent forms prior to participating in the study.

Four experiments were completed: (1) a reproducibility experiment, wherein a 2-frame measurement was made both before and after lifting and placing the probe at approximately the same location for three times $(n=19) ;(2)$ a stability experiment, wherein continuous data was collected for 10 frames ( 3.5 minutes) with subject breathing room air ( $n=24)$; (3) a maternal left tilt experiment wherein 4 frames of data were collected, first in supine and then in left-lateral decubitus position, to characterize hemodynamic changes related to increased maternal cardiac output and uterine perfusion ( $n=3)$; (4) a maternal hyperoxia experiment $(n=24)$, wherein the placenta was monitored continuously for 10 frames ( 3.5min) of baseline at room air, 20 frames ( 7 $\mathrm{min})$ of maternal hyperoxia $\left(100 \% \mathrm{FiO}_{2}\right)$, and 10 frames $(\sim 3.5 \mathrm{~min})$ of recovery at room air again. Note, a single "optical frame" corresponds to a measurement cycle through 11 light source-detector pairs and 3 wavelengths in $~ 21$ seconds.

In total, $\mathrm{n}=24$ subjects participated in this study. Detailed information about the subjects are provided in Extended Data Table 3. Note, data from two other subjects were excluded because their signals were either too small (tissue optical absorption coefficient was very large, $\mu_{a}>0.2 \mathrm{~cm}^{-1}$ ) or too unstable (due to large fluctuations during baseline period).

Measurement reproducibility was evaluated using the Intra-class Correlation Coefficient (ICC). We measured the hemoglobin properties multiple times at the same placental location in 19 subjects.

The stability test $(n=24)$ results were represented by the standard deviation (S.D.) during the continuous 10 frames measurements (Extended Data Fig. 2 (a)). Note, occasionally during data acquisition, substantial movement artifacts can occur causing a single frame to exhibit $>10 \%$ fluctuations in $\mathrm{StO}_{2}$, $\left[\mathrm{Hb}_{\mathrm{T}}\right]$, or $\left[\mathrm{HbO}_{2}\right]$ 
compared to the values of nearby frames. We identified these motion artifacts and filtered them out from the data.

To further validate the US/FD-DOS instrumentation and methodology in a physiologic context, we performed a left tilt experiment $(n=3)$. In this experiment, the impact of increased maternal cardiac output on placental oxygen hemoglobin properties were determined. Briefly, in each subject, we measured $\mathrm{StO}_{2},\left[\mathrm{Hb}_{\mathrm{T}}\right]$, and $\left[\mathrm{HbO}_{2}\right]$ for 4 frames both before and after the maternal tilt. Extended Data Fig. 2 (b) presents the mean values of $\mathrm{StO}_{2},\left[\mathrm{Hb}_{T}\right]$, and $\left[\mathrm{HbO}_{2}\right]$ for each subject, before and after the maternal tilt. The mean (S.D.) of relative increases (in percent) for these parameters due to the maternal tilt are $2.4(5.1), 8.4(3.2)$, and 10.9 (2.6) respectively. To calculate the mean (S.D.) of relative increases, we first determined the "before/after" difference in mean value of each parameter for each subject. Then we normalized this difference by the mean of the "before" value. Finally, we averaged these fractional changes across all 3 subjects (see Extended Data Figure 2 (b)). Although the number of subjects was very small, we observed a trend wherein both $\left[\mathrm{Hb}_{\mathrm{T}}\right]$ and $\left[\mathrm{HbO}_{2}\right]$ increased in the lateral position $\left(\mathrm{P}=0.046\right.$ and 0.018 respectively). StO $\mathrm{O}_{2}$ were comparable in the two states with $\mathrm{P}=0.507$. To calculate the $\mathrm{P}$ values, the relative hemoglobin properties were obtained by normalizing the "after" mean values to the "before" mean values, and paired t test analysis was applied to the relative "before/after" values.

The placental hemodynamic response to maternal hyperoxia was examined by monitoring the placental hemoglobin properties ([Hb], $\left.\left[\mathrm{HbO}_{2}\right],\left[\mathrm{Hb}_{\mathrm{T}}\right], \mathrm{StO}_{2}\right)$ before, during, and after maternal hyperoxia. After an initial baseline period, subjects were given $100 \% \mathrm{FiO}_{2}$ via facemask for $\sim 7$ minutes (20 frames). Concurrent US and FD-DOS data were acquired throughout the process. Overall $(n=24)$, the method easily resolved changes in placental blood oxygenation due to maternal hyperoxia. Note, occasionally during data acquisition, substantial movement artifacts can occur causing a single frame to exhibit $>10 \%$ fluctuations in $\mathrm{StO}_{2}$, $\left[\mathrm{Hb}_{\mathrm{T}}\right]$, or $\left[\mathrm{HbO}_{2}\right]$ compared to the values of nearby frames. Also, during the hyperoxia, occasionally, these artifacts caused a single frame to exhibit a dramatic drop in $\mathrm{StO}_{2}$ or $\left[\mathrm{HbO}_{2}\right](<95 \%$ of mean baseline). We identified these motion artifacts and filtered them out from the data.

We investigated potential associations between placental oxygen dynamics during maternal hyperoxia, (i.e., $\Delta \mathrm{StO}_{2}, \Delta \mathrm{Hb}_{\mathrm{T}}$, and $\Delta \mathrm{HbO}_{2}$ ) and the APO / MVM outcomes. For these analyses, the mean baseline $\mathrm{StO}_{2},\left[\mathrm{Hb}_{\mathrm{T}}\right]$, and $\left[\mathrm{HbO}_{2}\right]$ were calculated using the final 4 frames of the baseline period. $\Delta \mathrm{StO}_{2}, \Delta \mathrm{Hb} \mathrm{b}_{T}$, and $\Delta \mathrm{HbO}_{2}$ were defined as the difference between these mean baseline values and the "peak" values of the 4 -frame window during maternal 
hyperoxia wherein maximum $\mathrm{StO}_{2}$ occurred. Each subject $(\mathrm{N}=24)$ was then categorized into two groups based on pregnancy outcome: NPO or APO. We observed a significantly larger $\Delta \mathrm{StO}_{2}$ and $\Delta \mathrm{HbO}_{2}$ in response to maternal hyperoxia in the NPO group compared to a more blunted response in the APO group (see main text Fig. 4 (b)).

Similarly, when analyzing placental histopathology as the outcome of interest, we observed significant (large)

$\Delta \mathrm{StO}_{2}$ and $\mathrm{HHbO}_{2}$ in the NPP group compared to a blunted response in the MVM group (see main text Fig. 5 (b)). Wilcoxon rank sum tests were performed to calculate the $p$-values for comparison of different variables between NPO vs APO groups and NPP vs MVM groups.

We also analyzed the static and dynamic hemoglobin properties of the adipose and rectus/uterus layer from our three-layer reconstruction (Extended Data Table 4). (Note, due to very thin adipose or rectus/uterus layer thickness, 4 of the 24 subjects were processed with two-layer model reconstruction instead of three-layer model reconstruction; therefore, we excluded these 4 subjects in the statistical analysis of adipose or rectus/uterus layer.) The resulting data provide in vivo evidence demonstrating the variability of the optical properties of the overlying layers, thereby underscoring the importance of the multi-layer modeling to separate layer responses. FD-DOS/US is critical for quantitative capture of placenta response; without the multi-layer model and associated instrumentation, estimates of placenta response would be contaminated by signals from the other layers.

Uterine Artery Doppler Pulsatility Index (UtA PI). Each uterine artery was identified using a transabdominal C1-5 ultrasound probe (GE Healthcare) via power Doppler mapping. Pulsed wave Doppler was then used to obtain three similar consecutive waveforms. PI was defined as the difference between peak systolic and end diastolic velocities divided by the mean velocity. The mean PI of the two uterine arteries was used for analysis. No association was found between UtA PI and APO or MVM (main text Table 1 (c)). Furthermore, when including UtA PI as a covariate, the associations between $\Delta \mathrm{StO}_{2}$ and $\mathrm{HHbO}_{2}$ and our outcomes (i.e. APO and MVM) remained apparent (Extended Data Table 5).

Statistical Analysis. Statistical analysis was performed using MATLAB 2019a. Depending on the data-type, results are presented as mean (S.D.) and median (IQR). ICC in the reproducibility experiment was calculated by dividing the random effect variance by the total variance. P-values for studying correlations between different variables and placental dysfunction were obtained by two-sided Wilcoxon rank sum test, which is a nonparametric test for two populations when samples are independent. P values for studying correlations between nulliparity and 
1 placental dysfunction were obtained by two-sided Fisher's exact test. P-values for studying the before/after difference in the maternal left tilt experiment were calculated by two-sided paired sample t test analysis. Binary logistic regressions were also performed to study the correlation between APO/MVM and $\Delta \mathrm{StO}_{2}$ or $\Delta \mathrm{HbO}_{2}$ but with control of other variables: UtA PI, placental depth $(d)$, and pre-gravid BMI. We carried out this analysis for completeness with caveats that the sample size is small and that different pairs of variables might be partially correlated (and if so, that future inclusion of interactions in the statistical models is desirable). Extended Data Table 5 shows the resultant P-values of $\mathrm{SHbO}_{2}$ and $\mathrm{SStO}_{2}$ for prediction of APO or MVM from the binary logistic regression models. The results confirm that there remained a trend towards significant association between optically-derived hemodynamic properties and our outcomes of interest. For the future, a larger sample size will permit more sophisticated statistical analyses that explore the effects of possible confounding variables and that generate composite metrics with improved specificity and sensitivity.

\section{Data Availability}

The data that support the findings of this study are available in the main text of the paper and in its supplementary materials. In addition, all raw data are available from the corresponding author upon request.

\section{Code Availability}

The custom code employed for processing the optical data and for performing the statistical analysis are available online at https://github.com/LynnWong34. The LabVIEW code and simulation code are also available from the corresponding author upon request.

\section{References}

1. Burton, G. J., Fowden, A. L. \& Thornburg, K. L. Placental Origins of Chronic Disease. Physiol. Rev. 96, 1509-65 (2016).

2. Thornburg, K. L., O'Tierney, P. F. \& Louey, S. Review: The placenta is a programming agent for cardiovascular disease. Placenta 31 Suppl, S54-9 (2010).

3. Hodyl, N. A. et al. Child neurodevelopmental outcomes following preterm and term birth: What can the placenta tell us? Placenta 57, 79-86 (2017).

4. AIUM-ACR-ACOG-SMFM-SRU Practice Parameter for the Performance of Standard Diagnostic Obstetric Ultrasound Examinations. J. Ultrasound Med. 37, E13-E24 (2018). 
5. Turco, M. Y. et al. Trophoblast organoids as a model for maternal-fetal interactions during human placentation. Nature 564, 263-281 (2018).

6. Hemberger, M., Hanna, C. W. \& Dean, W. Mechanisms of early placental development in mouse and humans. Nature Reviews Genetics 21, 27-43 (2020).

7. Carter, A. M. Animal Models of Human Placentation - A Review. Placenta 28, (2007).

8. Schmidt, A., Morales-Prieto, D. M., Pastuschek, J., Fröhlich, K. \& Markert, U. R. Only humans have human placentas: Molecular differences between mice and humans. J. Reprod. Immunol. 108, 65-71 (2015).

9. Nye, G. A. et al. Human placental oxygenation in late gestation: experimental and theoretical approaches. J. Physiol. 596, 5523-5534 (2018).

10. Horsman, M. R., Mortensen, L. S., Petersen, J. B., Busk, M. \& Overgaard, J. Imaging hypoxia to improve radiotherapy outcome. Nature Reviews Clinical Oncology 9, 674-687 (2012).

11. You, W. et al. Hemodynamic Responses of the Placenta and Brain to Maternal Hyperoxia in Fetuses with Congenital Heart Disease by Using Blood Oxygen-Level Dependent MRI. Radiology 294, 141-148 (2020).

12. Logothetis, N. K. What we can do and what we cannot do with fMRI. Nature 453, 869-878 (2008).

13. Durduran, T., Choe, R., Yodh, A. G. \& Baker, W. B. Diffuse optics for tissue monitoring and tomography. Reports Prog. Phys. 73, (2010).

14. Yodh, A. G. \& Boas, D. A. Functional Imaging with Diffusing Light. in Biomedical Photonics Handbook: Biomedical Diagnostics 21 (2014).

15. Boas, D. A., O'Leary, M. A., Chance, B. \& Yodh, A. G. Scattering of diffuse photon density waves by spherical inhomogeneities within turbid media: Analytic solution and applications. Proc. Natl. Acad. Sci. U. S. A. 91, 4887-4891 (1994).

16. Waterhouse, D. J., Fitzpatrick, C. R. M., Pogue, B. W., O’Connor, J. P. B. \& Bohndiek, S. E. A roadmap for the clinical implementation of optical-imaging biomarkers. Nature Biomedical Engineering 3, 339-353 (2019).

17. Baker, W. B. et al. Continuous non-invasive optical monitoring of cerebral blood flow and oxidative metabolism after acute brain injury. J. Cereb. Blood Flow Metab. 39, 1469-1485 (2019).

18. Choe, R. et al. Transabdominal near infrared oximetry of hypoxic stress in fetal sheep brain in utero. Proc. Natl. Acad. Sci. 100, 12950-12954 (2003). 
19. Tromberg, B. J. et al. Predicting responses to neoadjuvant chemotherapy in breast cancer: ACRIN 6691 trial of diffuse optical spectroscopic imaging. Cancer Res. 76, 5933-5944 (2016).

20. Boas, D. A., Elwell, C. E., Ferrari, M. \& Taga, G. Twenty years of functional near-infrared spectroscopy: introduction for the special issue. Neuroimage 85, 1-5 (2014).

21. Yun, S. H. \& Kwok, S. J. J. Light in diagnosis, therapy and surgery. Nature Biomedical Engineering 1, 116 (2017).

22. Zhu, Q. et al. Breast Cancer: Assessing Response to Neoadjuvant Chemotherapy by Using US-guided Near-Infrared Tomography. Radiology 266, 433-442 (2013).

23. Eggebrecht, A. T. et al. Mapping distributed brain function and networks with diffuse optical tomography. Nat. Photonics 8, 448-454 (2014).

24. Konecky, S. D. et al. Imaging complex structures with diffuse light. Opt. Express 16, 5048 (2008).

25. Ntziachristos, V. Going deeper than microscopy: The optical imaging frontier in biology. Nature Methods 7, 603-614 (2010).

26. Kakogawa, J., Sumimoto, K., Kawamura, T., Minoura, S. \& Kanayama, N. Noninvasive monitoring of placental oxygenation by near-infrared spectroscopy. Am. J. Perinatol. 27, 463-468 (2010).

27. Hasegawa, J. et al. Evaluation of placental function using near infrared spectroscopy during fetal growth restriction. J. Perinat. Med. 38, 29-32 (2010).

28. Scholkmann, F. et al. A review on continuous wave functional near-infrared spectroscopy and imaging instrumentation and methodology. Neurolmage 85, 6-27 (2014).

29. Boas, D. A., Pitris, C. \& Ramanujam, N. Handbook of biomedical optics. Handbook of Biomedical Optics (2016). doi:10.1201/b10951

30. Choi, J. et al. Noninvasive determination of the optical properties of adult brain: near-infrared spectroscopy approach. J. Biomed. Opt. 9, 221 (2004).

31. Liebert, A. et al. Assessment of inflow and washout of indocyanine green in the adult human brain by monitoring of diffuse reflectance at large source-detector separation. J. Biomed. Opt. 16, 046011 (2011).

32. Pifferi, A. et al. New frontiers in time-domain diffuse optics, a review. J. Biomed. Opt. 21, 091310 (2016).

33. Wright, E. et al. Maternal Vascular Malperfusion and Adverse Perinatal Outcomes in Low-Risk Nulliparous Women. Obstet. Gynecol. 130, 1112-1120 (2017). 
34. Ernst, L. M. Maternal vascular malperfusion of the placental bed. APMIS 126, 551-560 (2018).

35. Liemert, A. Light diffusion in N-layered turbid media: frequency and time domains. J. Biomed. Opt. 15, 025003 (2010).

36. Ripoll, J. et al. Recovery of optical parameters in multiple-layered diffusive media: theory and experiments. J. Opt. Soc. Am. A 18, 821 (2001).

37. Schweiger, M. \& Arridge, S. The Toast++ software suite for forward and inverse modeling in optical tomography. J. Biomed. Opt. 19, 040801 (2014).

38. Lee, S. W. Y., Khaw, K. S., Kee, W. D. N., Leung, T. Y. \& Critchley, L. A. H. Haemodynamic effects from aortocaval compression at different angles of lateral tilt in non-labouring term pregnant women. Br. J. Anaesth. 109, 950-956 (2012).

39. O'Gorman, N., Tampakoudis, G., Wright, A., Wright, D. \& Nicolaides, K. H. Uterine artery pulsatility index at 12, 22, 32 and 36 weeks' gestation in screening for pre-eclampsia. Ultrasound Obstet. Gynecol. 47, 565-572 (2016).

40. Management of intrapartum fetal heart rate tracings. Obstetrics and Gynecology 116, 1232-1240 (2010).

41. Catov, J. M. et al. Neonatal outcomes following preterm birth classified according to placental features. Am. J. Obstet. Gynecol. 216, 411.e1-411.e14 (2017).

42. Liemert, A. Light diffusion in N-layered turbid media: steady-state domain. J. Biomed. Opt. 15, 025003 (2010).

43. Haskell, R. C. et al. Boundary conditions for the diffusion equation in radiative transfer. J. Opt. Soc. Am. A. Opt. Image Sci. Vis. 11, 2727-41 (1994).

44. Jacques, S. L. Optical properties of biological tissues: A review (Physics in Medicine and Biology (2013) 58). Phys. Med. Biol. 58, 5007-5008 (2013).

45. Hansen, P. C. The L-curve and its use in the numerical treatment of inverse problems.

46. Ugray, Z. et al. Scatter search and local NLP solvers: A multistart framework for global optimization. INFORMS J. Comput. 19, 328-340 (2007).

47. Gestational Hypertension and Preeclampsia: ACOG Practice Bulletin, Number 222. Obstet. Gynecol. 135, e237-e260 (2020).

48. Fenton, T. R. \& Kim, J. H. A systematic review and meta-analysis to revise the Fenton growth chart for 
preterm infants. BMC Pediatr. 13, (2013).

2 49. Khong, T. Y. et al. Sampling and definitions of placental lesions Amsterdam placental workshop group consensus statement. in Archives of Pathology and Laboratory Medicine 140, 698-713 (College of American Pathologists, 2016).

\section{Acknowledgements}

6 The work was supported by the NIH U01HD087180. J.M.C. was partially supported by NIH P41EB015893. T.K.

7 was partially supported by NIH F31HD085731 and NIH T32HL007915. W.B. was partially supported by

8 R01NS113945. AGY acknowledges partial support from NIH R01NS060653 and NIH P41EB015893. We

9 gratefully acknowledge useful discussions with Daniel Licht, Brian White, Jerome Strauss, Yi Hong Ong for their

10 advice and support as well as the clinic research coordinators of the Maternal and Child Health Research Center

11 at Perelman School of Medicine, University of Pennsylvania.

\section{Author contributions}

13 L.W., A.G.Y., and N.S. designed the study. L.W. and T.K. developed the instrument with assistance of W.B.B., 14 K.A., L.H., D.R.B., and V.K. L.W. and J.M.C. developed the three-layer reconstruction algorithm and conducted 15 the computer simulations. L.W. and T. K. performed phantom experiments with help of W.B. and L.H. A.K. 16 designed the optical probe with input from L.W. and W.B.B. L.W. collected and analyzed the optical data. S.P. 17 and N.S. advised on human subject data interpretation. N.S. collected and analyzed the ultrasound data. R.L.L. performed placental histopathologic analysis. L.W., A.G.Y., and N.S. wrote the paper with input from all authors.

\section{Competing Interests statement}

The authors declare no competing interests. 
Extended Data Table 1 Two-layer phantom experiment results (785nm)

\begin{tabular}{|c|c|c|c|c|c|c|c|c|c|c|c|c|c|c|c|c|c|}
\hline \multicolumn{9}{|c|}{ Absorption-titration experiment } & \multicolumn{9}{|c|}{ Depth-changing experiment } \\
\hline$d$ & & EXP & MEA & Err & & EXP & MEA & Err & $d$ & & EXP & MEA & Err & & EXP & MEA & Err \\
\hline \multirow{2}{*}{$\begin{array}{c}3 \\
c n\end{array}$} & $\mu_{a, t}$ & 0.08 & 0.08 & $+6 \%$ & $\mu_{s, t}^{\prime}$ & 9.1 & 8.9 & $-3 \%$ & \multirow{2}{*}{$\begin{array}{l}1.5 \\
\mathrm{~cm}\end{array}$} & $\mu_{a, t}$ & 0.13 & 0.12 & $-4 \%$ & $\mu_{s, t}^{\prime}$ & 9.1 & 9.1 & $0 \%$ \\
\hline & $\mu_{a, b}$ & 0.10 & 0.11 & $+7 \%$ & $\mu_{s, b}^{\prime}$ & 5.0 & 5.8 & $+15 \%$ & & $\mu_{a, b}$ & 0.10 & 0.11 & $+7 \%$ & $\mu_{s, b}^{\prime}$ & 5.0 & 5.8 & $+15 \%$ \\
\hline \multirow{2}{*}{$\begin{array}{c}3 \\
\mathrm{~cm}\end{array}$} & $\mu_{a, t}$ & 0.10 & 0.10 & $-5 \%$ & $\mu_{s, t}^{\prime}$ & 9.1 & 8.9 & $-2 \%$ & \multirow{2}{*}{2.5} & $\mu_{a, t}$ & 0.13 & 0.12 & $-2 \%$ & $\mu_{s, t}^{\prime}$ & 9.1 & 8.8 & $-4 \%$ \\
\hline & $\mu_{a, b}$ & 0.10 & 0.10 & $-3 \%$ & $\mu_{s, b}^{\prime}$ & 5.0 & 5.2 & $+4 \%$ & & $\mu_{a, b}$ & 0.10 & 0.10 & $+0 \%$ & $\mu_{s, b}^{\prime}$ & 5.0 & 5.0 & $-1 \%$ \\
\hline \multirow{2}{*}{$\begin{array}{c}3 \\
\mathrm{~cm}\end{array}$} & $\mu_{a, t}$ & 0.13 & 0.12 & $-8 \%$ & $\mu_{s, t}^{\prime}$ & 9.1 & 9.1 & $+0 \%$ & \multirow{2}{*}{$\begin{array}{c}3 \\
\mathrm{~cm}\end{array}$} & $\mu_{a, t}$ & 0.13 & 0.12 & $-8 \%$ & $\mu_{s, t}^{\prime}$ & 9.1 & 9.1 & $+0 \%$ \\
\hline & $\mu_{a, b}$ & 0.10 & 0.10 & $-2 \%$ & $\mu_{s, b}^{\prime}$ & 5.0 & 5.2 & $+4 \%$ & & $\mu_{a, b}$ & 0.10 & 0.10 & $-2 \%$ & $\mu_{s, b}^{\prime}$ & 5.0 & 5.2 & $+4 \%$ \\
\hline
\end{tabular}

\begin{tabular}{|c|c|c|c|c|c|c|c|c|c|c|}
\hline & \multicolumn{2}{|c|}{$\mathrm{StO}_{2}(\%)$} & \multicolumn{2}{c|}{$\left[\mathrm{Hb}_{T}\right](\mu \mathrm{M})$} & \multicolumn{2}{c|}{$\mu_{s}^{\prime}(785)\left(\mathrm{cm}^{-1}\right)$} & $\mu_{s}^{\prime}(808)\left(\mathrm{cm}^{-1}\right)$ & \multicolumn{2}{c|}{$\mu_{s}^{\prime}(830)\left(\mathrm{cm}^{-1}\right)$} \\
\hline & EXP & MEA & EXP & MEA & EXP & MEA & EXP & MEA & EXP & MEA \\
\hline Adipose & 78.6 & 75.2 & 7.5 & 9.4 & 9.8 & 9.2 & 9.5 & 9.2 & 9.3 & 8.9 \\
\hline Rectus/Uterus & 68.1 & 70.0 & 60.1 & 54.5 & 6.1 & 6.6 & 5.9 & 6.4 & 5.7 & 6.2 \\
\hline Placenta & 72.1 & 71.6 & 68.1 & 69.8 & 4.4 & 6.5 & 4.2 & 6.3 & 4.1 & 6.2 \\
\hline \multicolumn{3}{|c|}{ Note: EXP denotes expected value; MEA denotes measured value. } \\
\hline
\end{tabular}

\begin{tabular}{|c|c||c|c|}
\hline \multicolumn{1}{|c|}{ Parameter } & Median (IQR) & Parameter & Median (IQR) \\
\hline Nulliparity & $29.2 \%$ & Maternal age at delivery (years) & $27(24.0,33.0)$ \\
\hline $\begin{array}{c}\text { Pre-gravid BMI }\left(\mathrm{kg} / \mathrm{m}^{2}\right) \\
\text { Gestational age at measurement } \\
\text { (weeks) }\end{array}$ & $\begin{array}{c}27.7(24.6,30.2) \\
(32.9,35.44)\end{array}$ & $\begin{array}{c}\text { Delivery BMI }\left(\mathrm{kg} / \mathrm{m}^{2}\right) \\
\text { Latency from measurement to } \\
\text { delivery (weeks) }\end{array}$ & $31.2(28.2,34.5)$ \\
\hline $\begin{array}{c}\text { Anterior placenta depth (cm) } \\
\text { Birth weight }(\mathrm{kg})\end{array}$ & $2.7(2.3,3.3)$ & Gestational age at delivery (weeks) & $38.3(37.3,39.3)$ \\
\hline Gestational hypertension (GHTN) & $3.0(2.6,3.3)$ & Intrauterine growth restriction (IUGR) & $4.2 \%$ \\
\hline Note: Parameters are summarized as median (IQR) or by percentage. & $16.7 \%$ \\
\hline
\end{tabular}


Extended Data Table 4 Baseline/Dynamic hemoglobin properties of adipose, rectus/uterus, placenta layers $\left(n=20^{\star}\right)$

\begin{tabular}{|c|c|c|c|c|c|c|}
\hline $\mathrm{n}=20^{*}$ & $\mathrm{StO}_{2}(\%)$ & {$\left[\mathrm{Hb}_{\mathrm{T}}\right](\mu \mathrm{M})$} & {$\left[\mathrm{HbO}_{2}\right](\mu \mathrm{M})$} & $\Delta \mathrm{StO}_{2}(\%)$ & $\Delta \mathrm{Hb}_{T}(\mu \mathrm{M})$ & $\Delta \mathrm{HbO}_{2}(\mu \mathrm{M})$ \\
\hline Adipose & $81.4(75.9,88.5)$ & $13.1(8.5,17.6)$ & $10.6(6.2,14.7)$ & $6.9(3.4,12.0)$ & $0.1(-1.1,1.6)$ & $1.2(0.2,2.5)$ \\
\hline Rectus/uterus & $78.1(71.8,85.7)$ & $25.3(20.4,32.5)$ & $18.2(15.5,25.4)$ & $8.2(4.5,12.3)$ & $0.3(-1.1,1.9)$ & $2.2(1.7,2.9)$ \\
\hline Placenta & $72.8(67.5,77.0)$ & $27.0(22.0,33.6)$ & $20.6(14.2,24.6)$ & $6.7(5.8,8.9)$ & $-0.2(-0.7,0.7)$ & $1.7(1.2,2.8)$ \\
\hline
\end{tabular}

3

Extended Data Table 5 Binary logistic regression with two independent variables

\begin{tabular}{|c|c|c|c|c|c|c|c|c|}
\hline \multirow{6}{*}{$\begin{array}{l}N \\
= \\
24\end{array}$} & \multicolumn{4}{|c|}{ APO } & \multicolumn{4}{|c|}{ MVM } \\
\hline & \multicolumn{2}{|c|}{$\Delta \mathrm{StO}_{2}(\%)$} & \multicolumn{2}{|c|}{$\Delta H_{b} O_{2}(\mu M)$} & \multicolumn{2}{|l|}{$\Delta \mathrm{StO}_{2}(\%)$} & \multicolumn{2}{|c|}{$\Delta H b O_{2}(\mu M)$} \\
\hline & with control for & $P$ & with control for & $P$ & with control for & $\mathrm{P}$ & with control for & $P$ \\
\hline & UtA PI & 0.096 & UtA PI & 0.046 & UtA PI & 0.075 & UtA PI & 0.035 \\
\hline & $d(\mathrm{~cm})$ & 0.073 & $d(\mathrm{~cm})$ & 0.067 & $d(\mathrm{~cm})$ & 0.078 & $d(\mathrm{~cm})$ & 0.024 \\
\hline & $\mathrm{BMI}$ & 0.064 & BMI & 0.070 & BMI & 0.066 & $\mathrm{BMI}$ & 0.049 \\
\hline
\end{tabular}




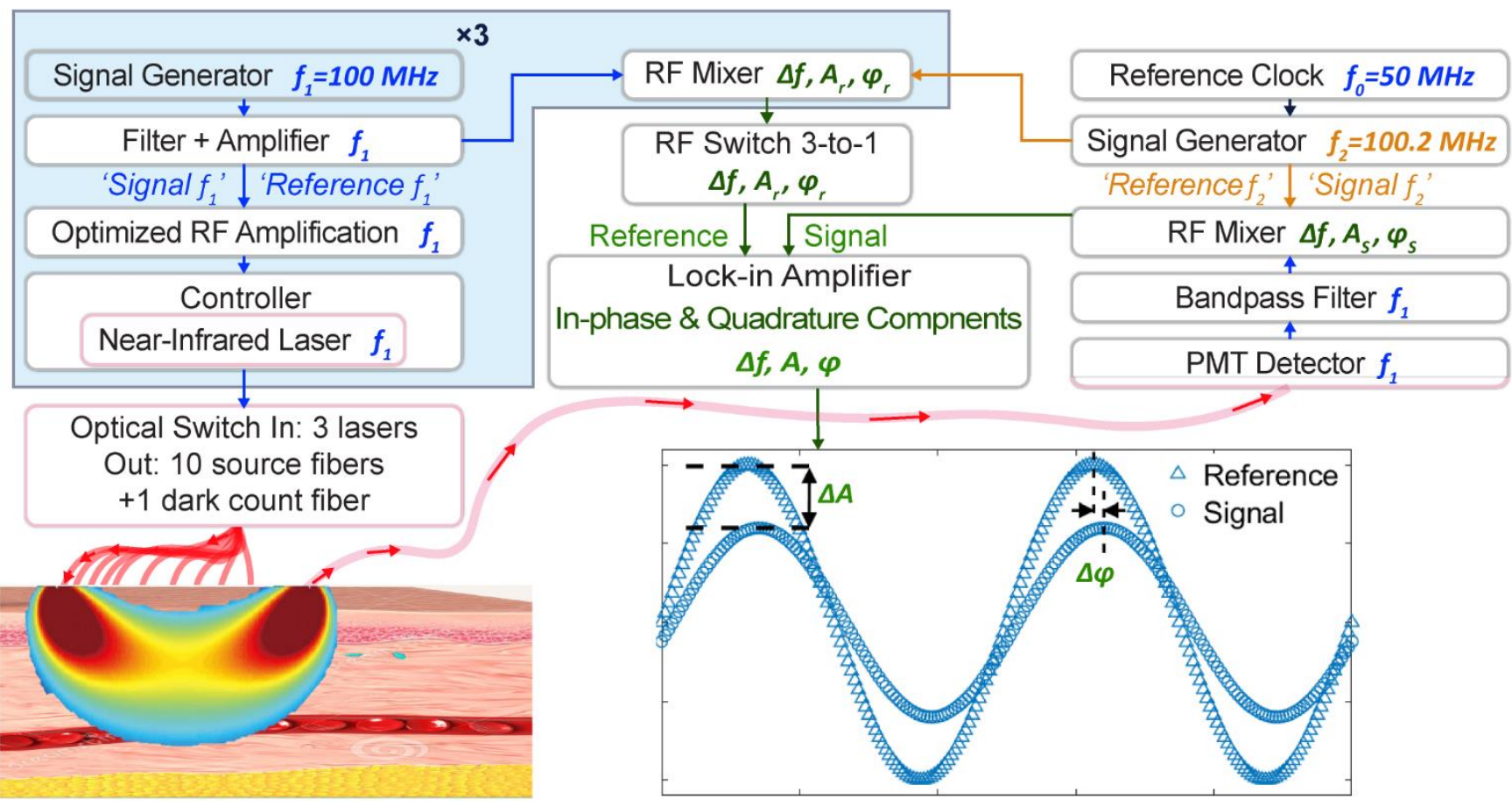

2 Extended Data Fig. 1: Detailed schematic of custom heterodyne FD-DOS instrument.

3

4 
(a) Mean (S.D.) of placental $\left[\mathrm{StO}_{2}\right],\left[\mathrm{Hb}_{\mathrm{T}}\right]$, and $\left[\mathrm{HbO}_{2}\right]$ vs $\mathrm{GA}$ derived from stability test measurements
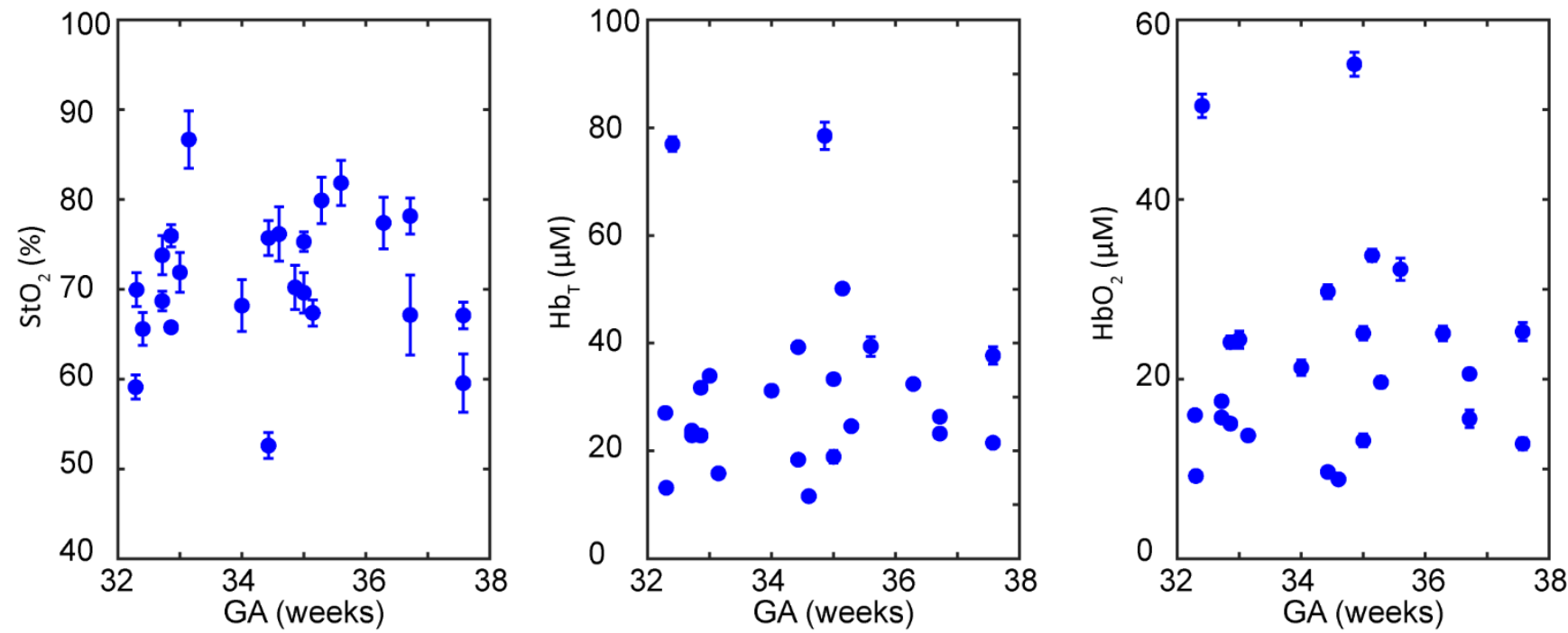

(b) Placental $\mathrm{StO}_{2^{\prime}}\left[\mathrm{Hb}_{\mathrm{T}}\right]$, and $\left[\mathrm{HbO}_{2}\right]$ of individual subject before and after maternal left tilt
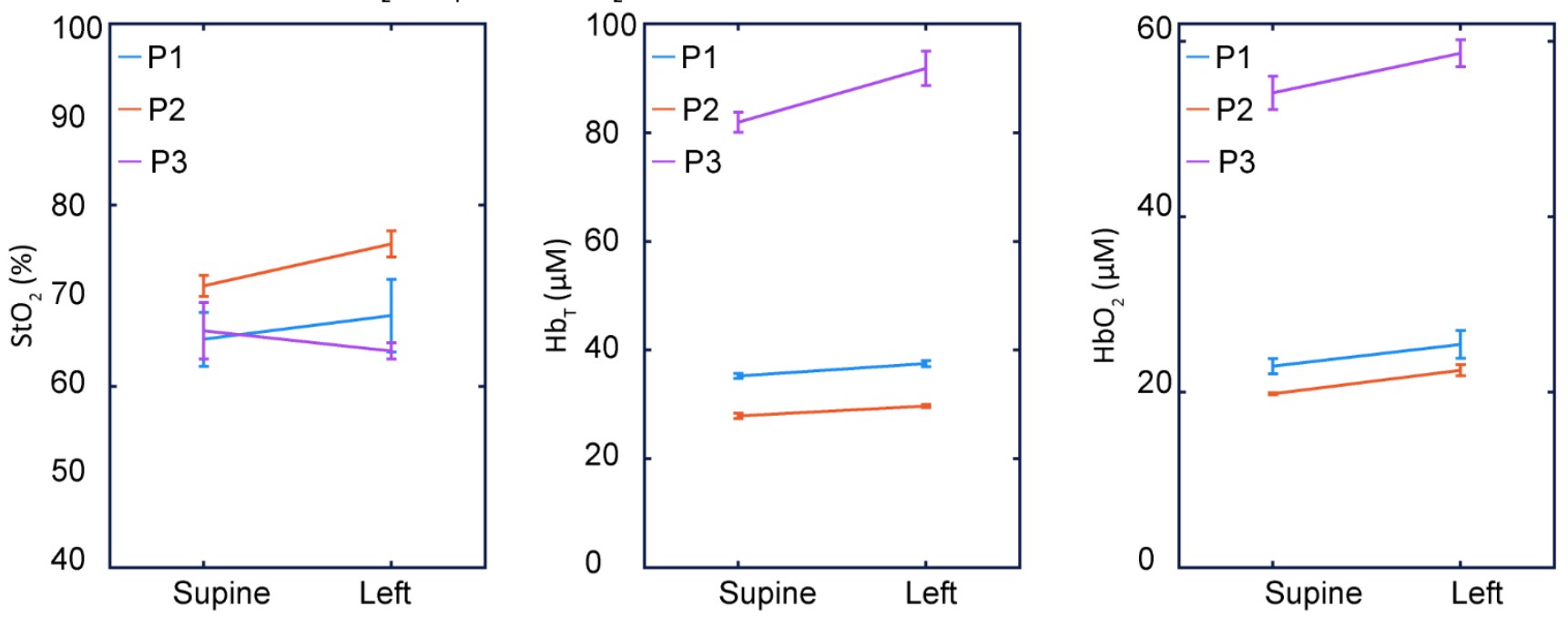

2 Extended Data Fig. 2: Placental hemoglobin properties during stability test measurements and maternal

3 left tilt experiment. (a) Mean (S.D.) of placental $\mathrm{StO}_{2},\left[\mathrm{Hb}_{\mathrm{T}}\right],\left[\mathrm{HbO}_{2}\right]$ versus gestational stage (GA) derived from

4 the stability test measurements (error bar indicates S.D.). (b) Placental $\mathrm{StO}_{2},\left[\mathrm{Hb}_{\mathrm{T}}\right],\left[\mathrm{HbO}_{2}\right]$ of three individual

5 subjects before/after maternal left tilt (error bar indicates S.D.). 


\section{Figures}

(a) Schematic of hybrid FD-DOS/US placenta instrumentation system

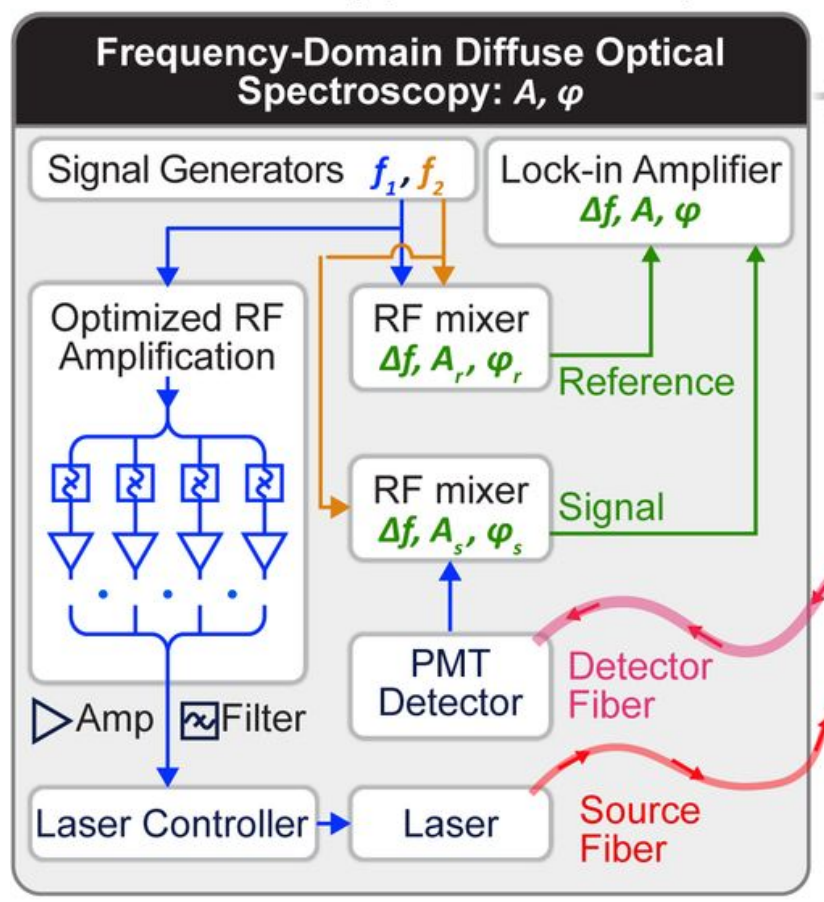

\section{Optical Reconstruction of Placental StO ${ }_{2},\left[\mathrm{Hb}_{T}\right],\left[\mathrm{HbO}_{2}\right]$}

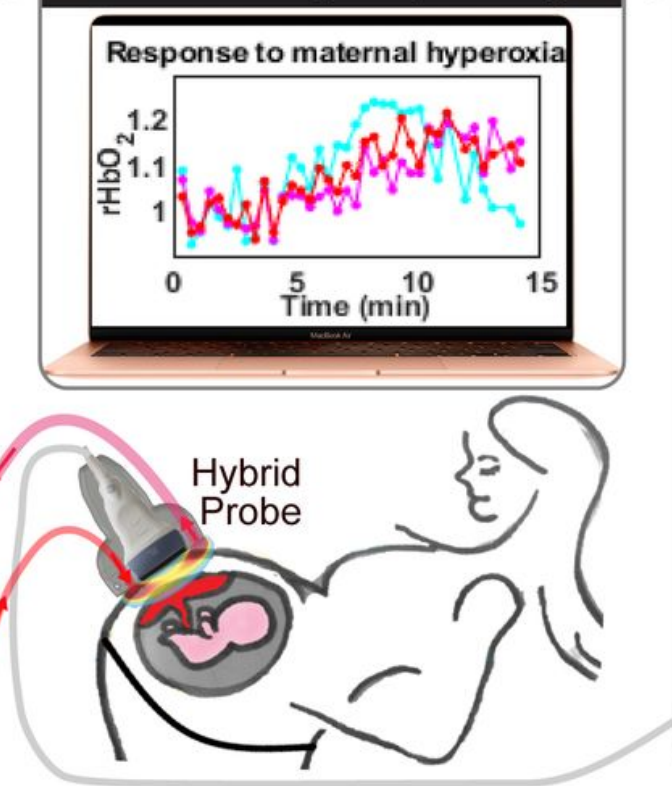

Ultrasound Image Segmentation: $d_{1}, d_{2}, d_{3}$
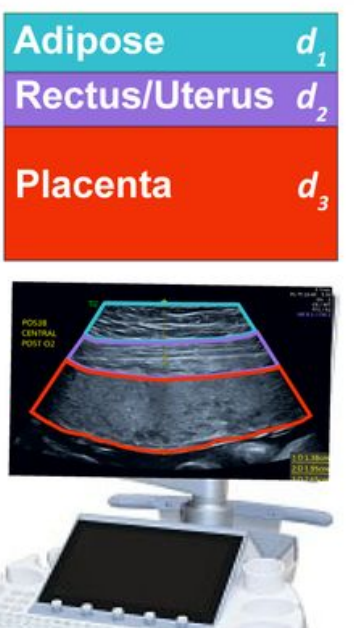

Ultrasound (b) Integration of optical and US instrumentation into single probe head

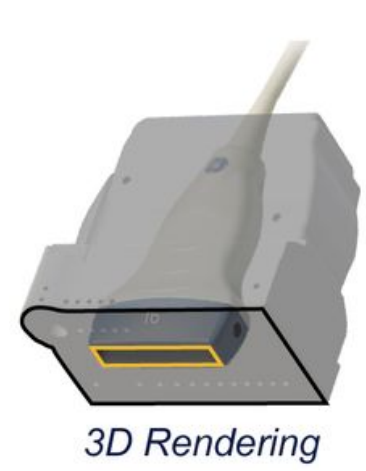

- Source Fiber

Detector Fiber

Ultrasound Probe

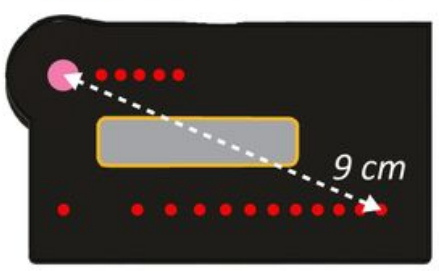

Hybrid Probe Schematic (c) US image segmentation and corresponding three-layer model for optical image reconstruction
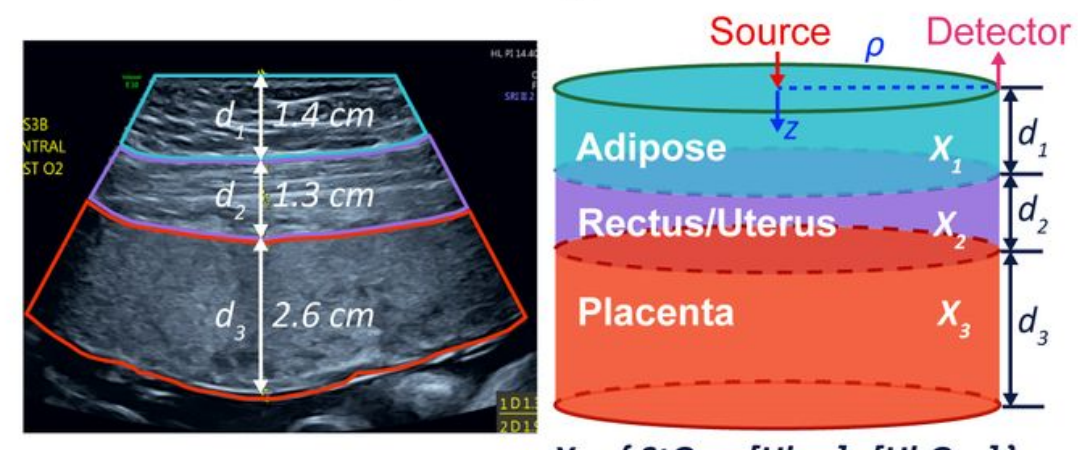

$X_{i}=\left\{\mathrm{StO}_{2, i},\left[\mathrm{Hb}_{\tau, i}\right],\left[\mathrm{HbO}_{2, i}\right]\right\}$

Figure 1

Integrated Frequency-Domain Diffuse Optical Spectroscopy/Ultrasound (FD-DOS/US) placenta instrumentation and three-layer modeling. (a) Schematic of the hybrid FD-DOS/US instrumentation showing laser amplitude modulation electronics, tissue light transmission, PMT detection, heterodyne mixing for frequency down-conversion, lock-in detection of light wave amplitude/phase, simultaneous US image segmentation, and optical image reconstruction of placental hemoglobin properties. (b) 3D rendering and bottom-view of probe. 
(a) Three-layer optical image reconstruction protocol

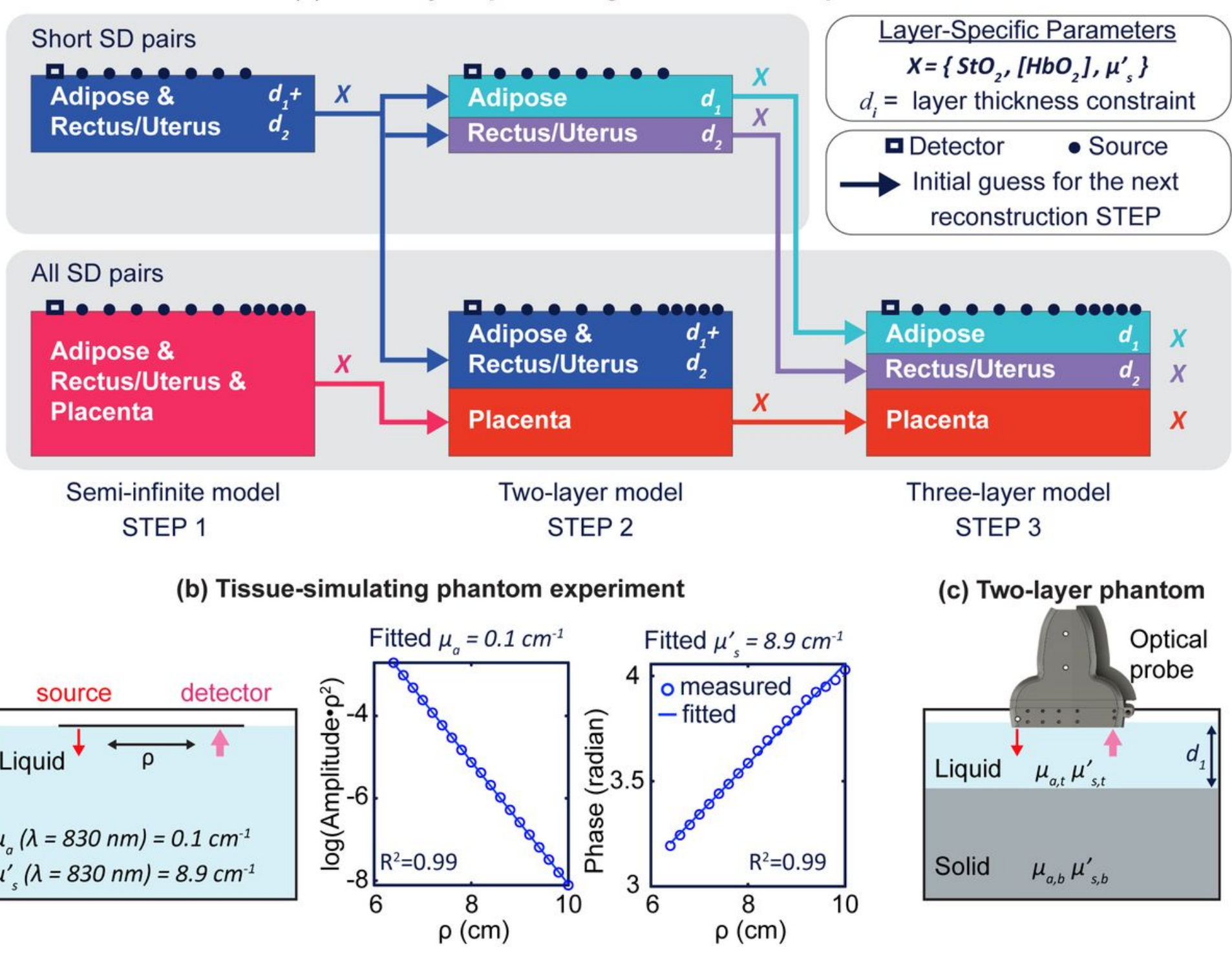

Figure 2

Three-layer model reconstruction algorithm and phantom validation experiments. (a) Three-layer model initialization and reconstruction procedure. (b) Tissue-simulating phantom experiment for validating accuracy and depth sensitivity. A translation stage moves the source fiber across the phantom for measurements at SDSs ranging from 6.2 to $10 \mathrm{~cm}$. Nonlinear semi-infinite fitting is performed for each wavelength to reconstruct phantom optical properties. (c) Two-layer phantom experiment schematic. 
(a) Placental $\mathrm{StO}_{2}$ change during maternal hyperoxia - exemplary case

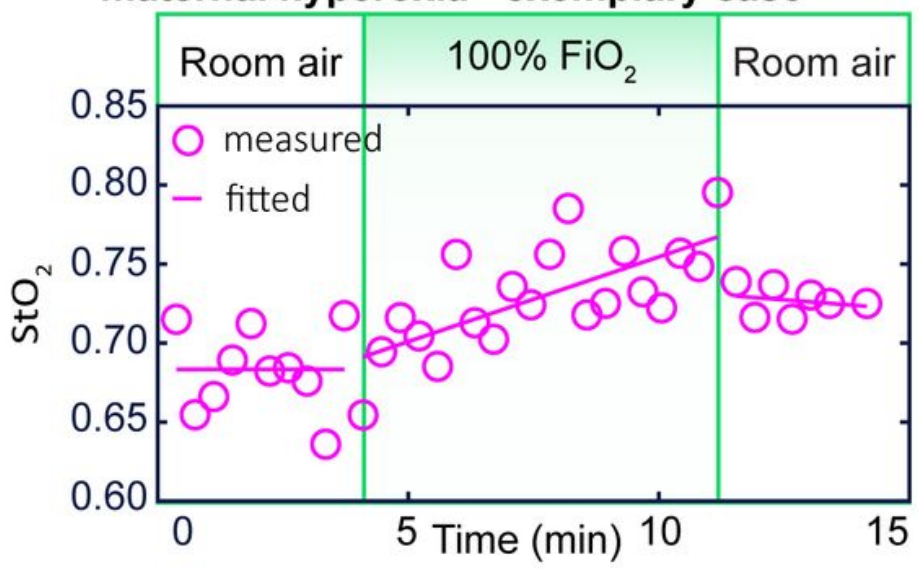

(c) Averaged placental hemodynamic responses to maternal hyperoxia of NPO group ( $n=15)$

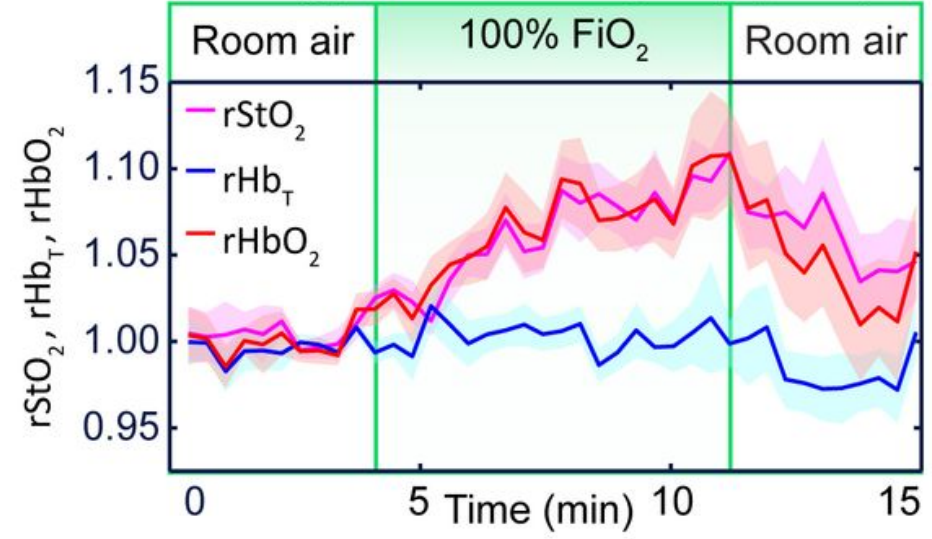

(e) Averaged placental hemodynamic responses to maternal hyperoxia of NPP group $(n=16)$

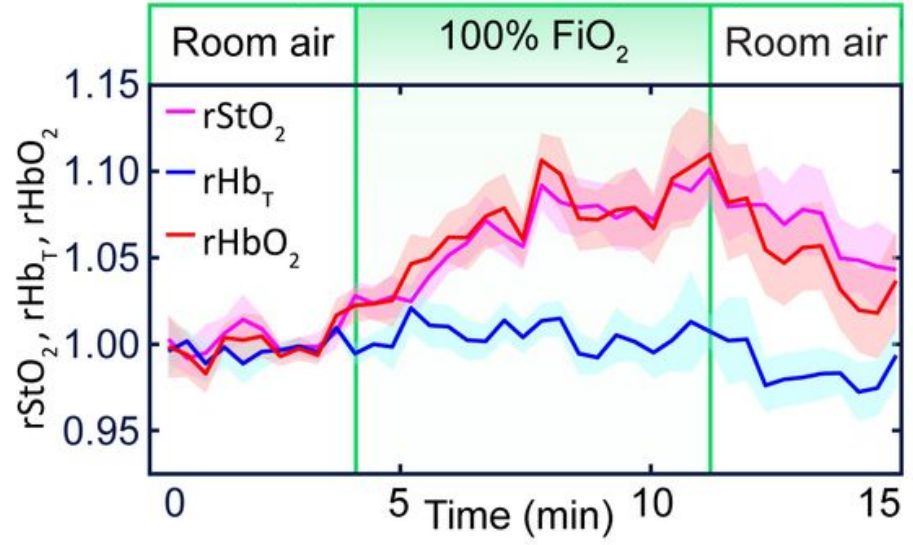

(b) Placental $\mathrm{HbO}_{2}$ change during maternal hyperoxia - exemplary case

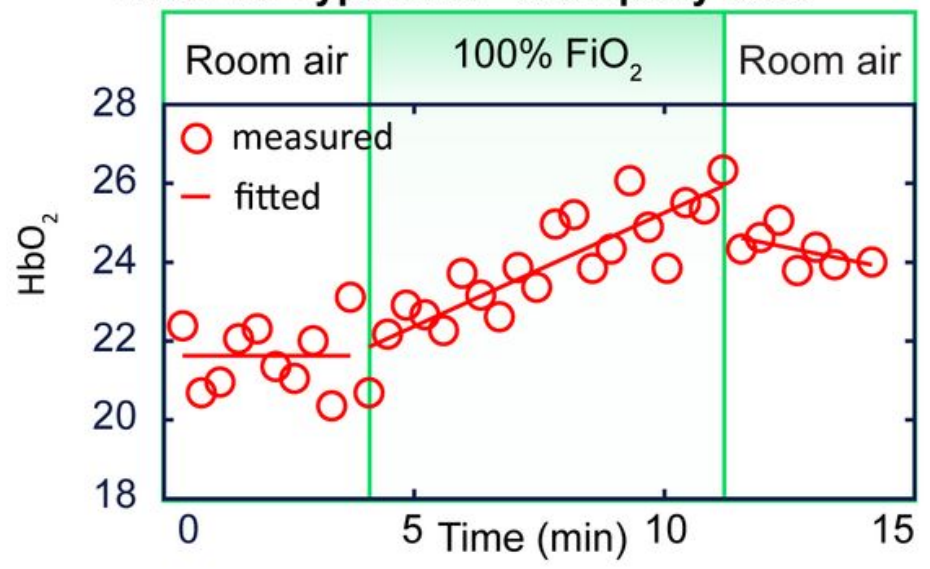

(d) Averaged placental hemodynamic responses to maternal hyperoxia of APO group $(n=9)$

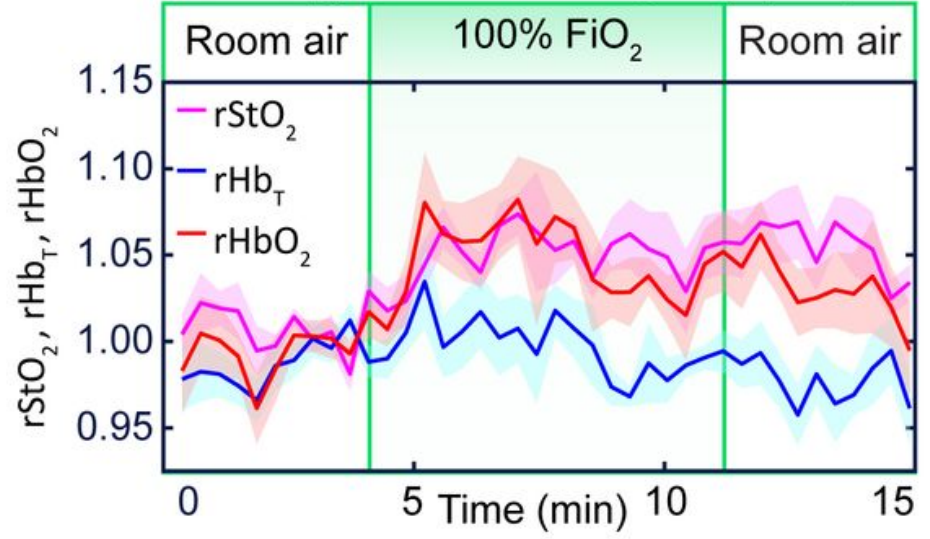

(f) Averaged placental hemodynamic responses to maternal hyperoxia of MVM group $(n=8)$

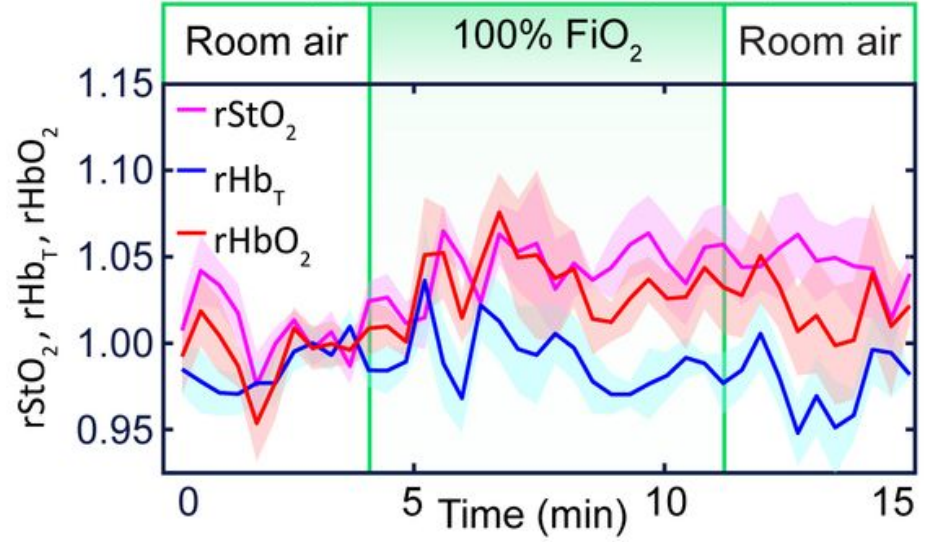

Figure 3

Continuous monitoring of placental hemoglobin properties during maternal hyperoxia. (a), (b): Exemplary

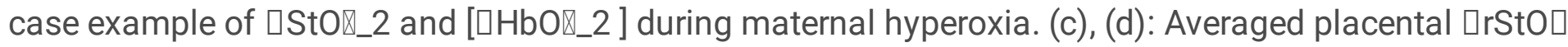

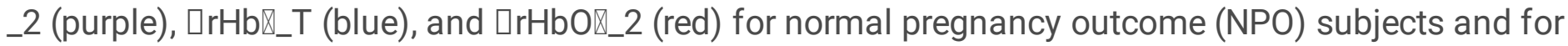

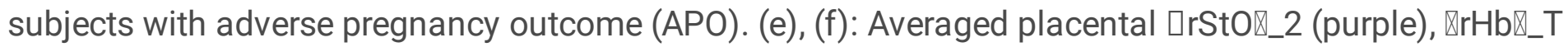
(blue), $\mathrm{\square rHbO} \unrhd_{-} 2$ (red) for normal placental pathology (NPP) subjects and for subjects with Maternal Vascular Malperfusion (MVM). Shaded regions represent standard error. Notice, the cohort averaged 


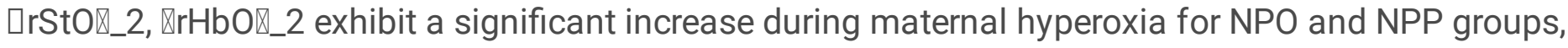
but a blunted response for APO and MVM groups.

(A) Placental hemoglobin properties during baseline for NPO and APO pregnancies
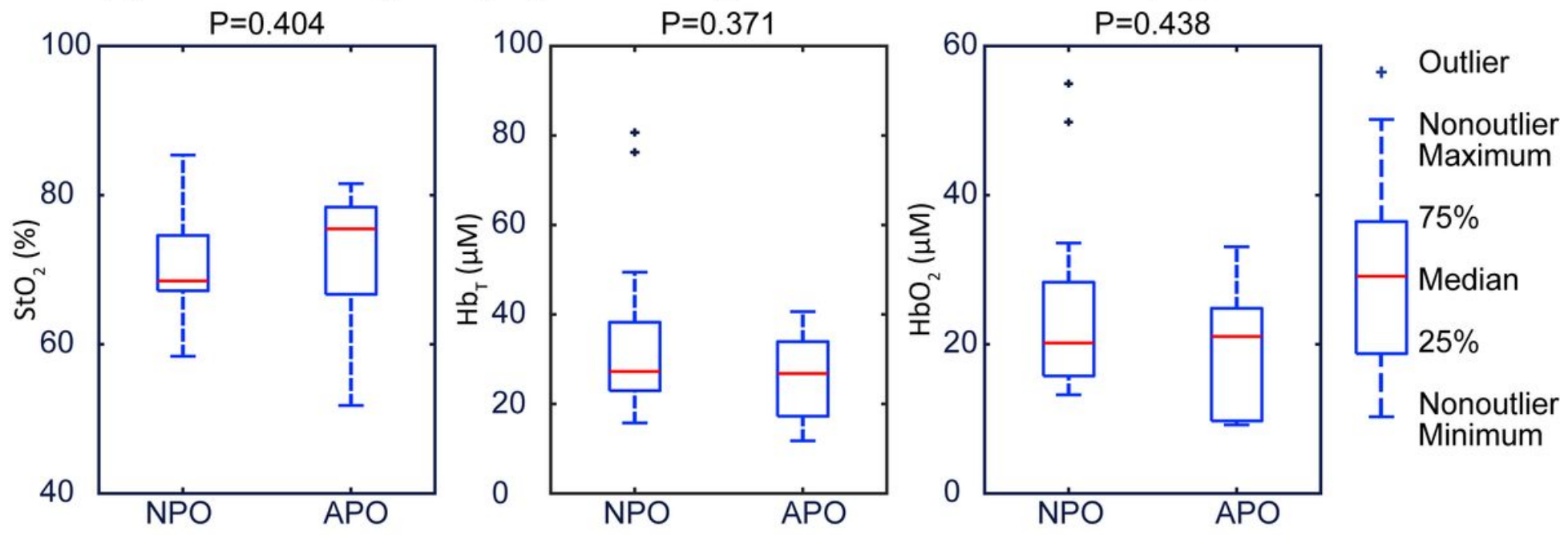

(B) Placental hemoglobin changes during maternal hyperoxia for NPO and APO pregnancies
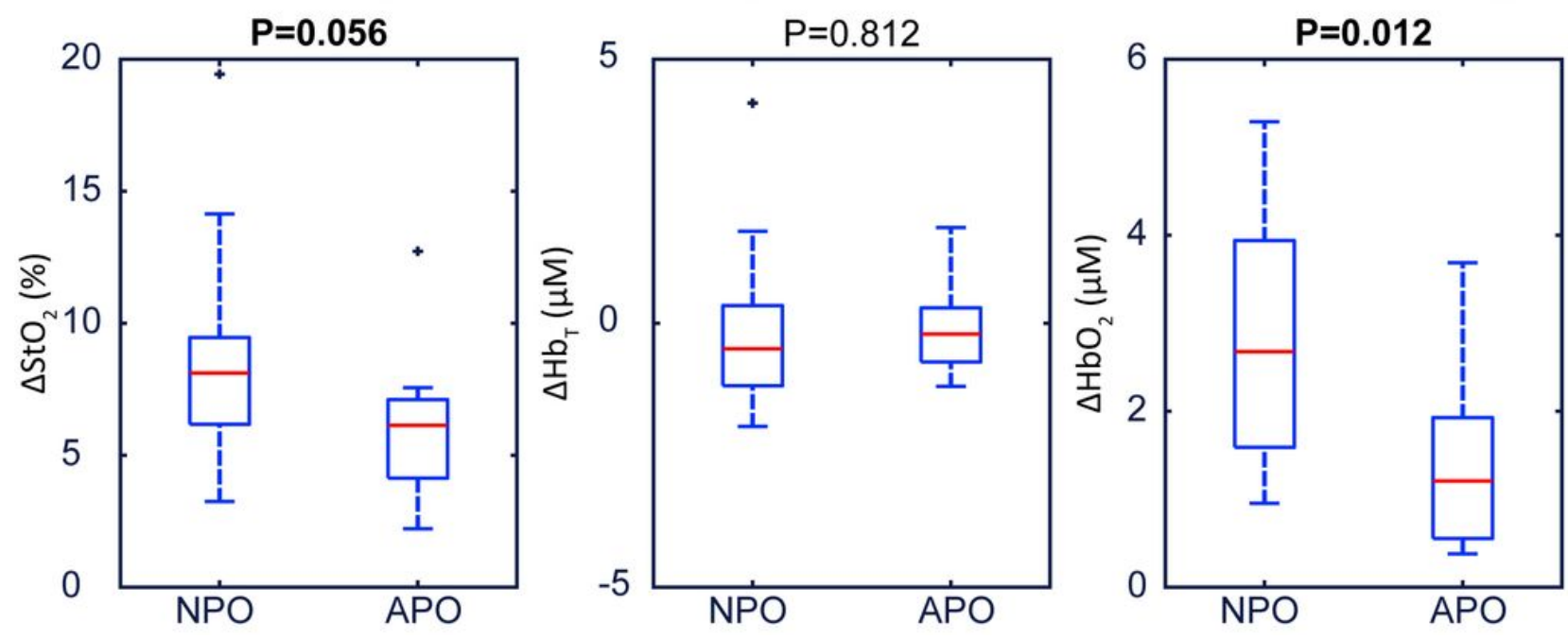

Figure 4

Boxplots of static and dynamic placental hemoglobin properties during maternal hyperoxia for subjects with normal pregnancy outcome (NPO) $(n=15)$ and Adverse Pregnancy Outcome (APO) ( $n=9)$. (a)

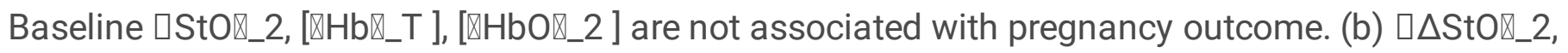
$\square \Delta \mathrm{Hb} \otimes \_\mathrm{T}, \square \Delta \mathrm{HbO} \_\_2$ during maternal hyperoxia. $\square \Delta \mathrm{StO} \mathbb{Q} \_2$ and $\square \Delta \mathrm{HbO} \_\_2$ exhibit clear separation amongst subjects with NPO versus APO. P values are calculated by Wilcoxon rank sum test. 
(a) Placental hemoglobin properties during baseline for NPP and MVM pregnancies
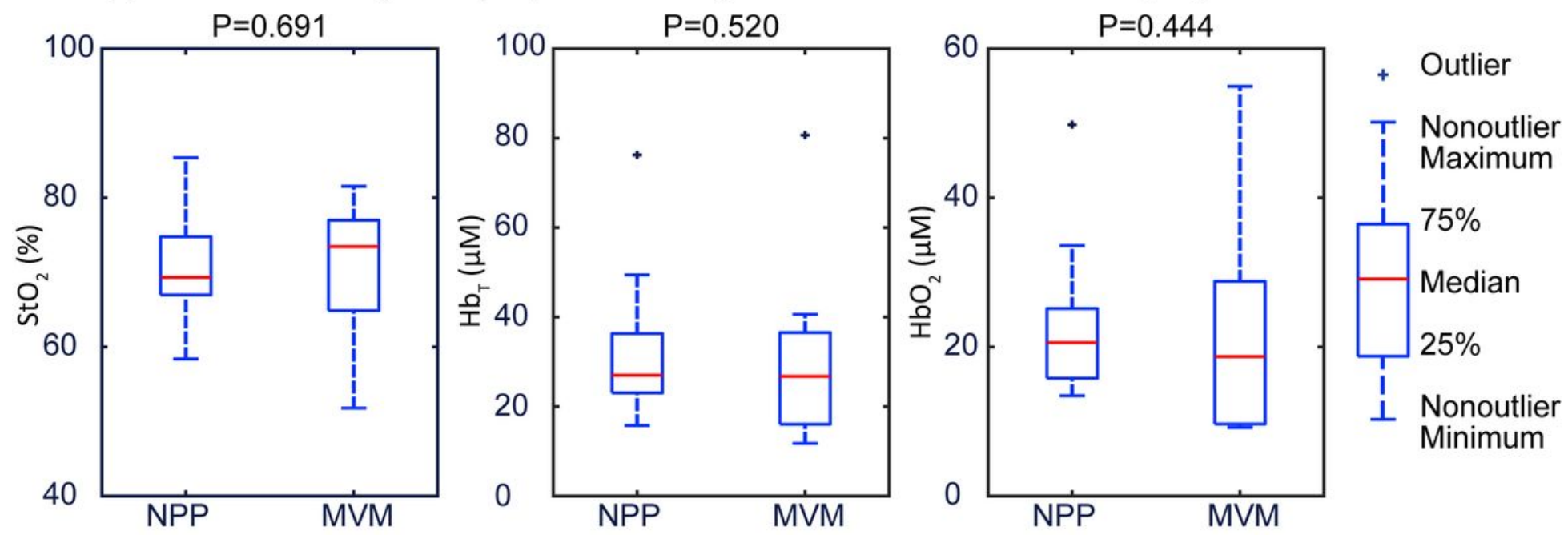

(b) Placental hemoglobin changes during maternal hyperoxia for NPP and MVM pregnancies
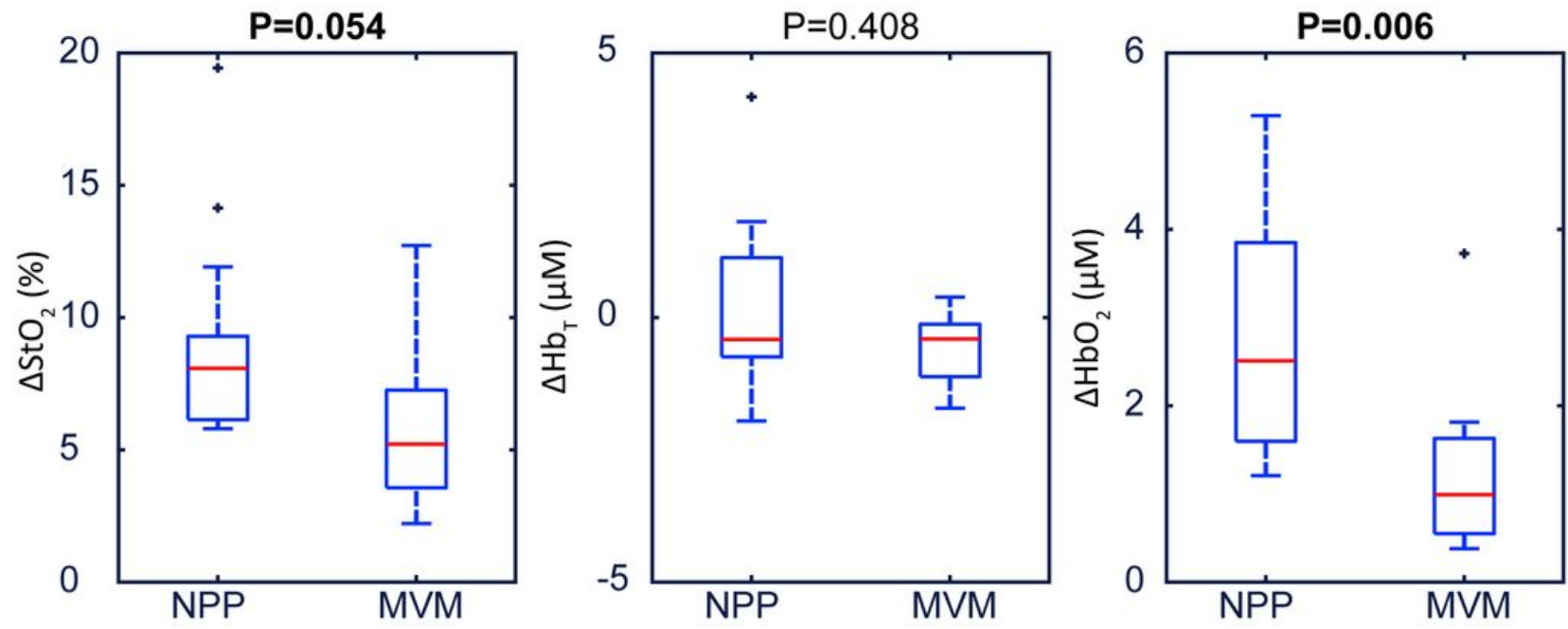

Figure 5

Boxplots of static and dynamic placental hemoglobin properties during maternal hyperoxia for subjects with normal placental pathology (NPP) $(n=16)$ and Maternal Vascular Malperfusion (MVM) $(n=8)$. (a)

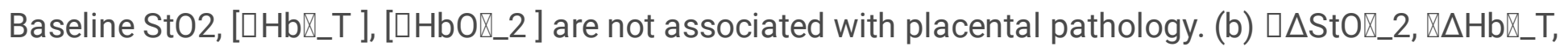

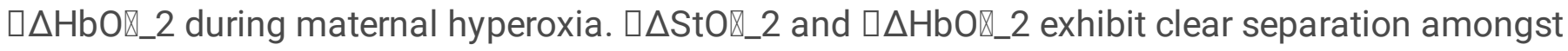
subjects with NPP versus MVM. P values are calculated by Wilcoxon rank sum test.

\section{Supplementary Files}

This is a list of supplementary files associated with this preprint. Click to download.

- P35V3Data.zip

- ExtendedDataFigure1Noninvasiveinvivoopticalmonitoringofhumanplacentaloxygenation.jpg

- ExtendedDataFigure2Noninvasiveinvivoopticalmonitoringofhumanplacentaloxygenation.jpg 